\title{
Theory of a Weak-Link Superconductor-Ferromagnet Josephson Structure
}

\author{
J. Gelhausen ${ }^{1,2}$ and M. Eschrig ${ }^{2}$ \\ ${ }^{1}$ Institut für Theoretische Physik, Universität zu Köln, 50937 Cologne, Germany \\ ${ }^{2}$ Department of Physics, Royal Holloway, University of London, Egham, Surrey TW20 0EX, United Kingdom
}

(Dated: February 28, 2022)

\begin{abstract}
We propose a model for the theoretical description of a weak-link Josephson junction, in which the weak link is spin-polarized due to proximity to a ferromagnetic metal $(\mathrm{S}-(\mathrm{F} \mid \mathrm{S})-\mathrm{S})$. Employing Usadel transport theory appropriate for diffusive systems, we show that the weak link is described within the framework of Andreev circuit theory by an effective self-energy resulting from the implementation of spin-dependent boundary conditions. This leads to a considerable simplification of the model, and allows for an efficient numerical treatment. As an application of our model, we show numerical calculations of important physical observables such as the local density of states, proximity-induced minigaps, spin-magnetization, and the phase and temperature-dependence of Josephson currents of the $\mathrm{S}-(\mathrm{F} \mid \mathrm{S})-\mathrm{S}$ system. We discuss multi-valued current-phase relationships at low temperatures as well as their crossover to sinusoidal form at high temperatures. Additionally, we numerically treat (S-F-S) systems that exhibit a magnetic domain wall in the $\mathrm{F}$ region and calculate the temperaturedependence of the critical currents.
\end{abstract}

PACS numbers: 72.25.-b, 72.25.MK, 74.45.+c, 74.78.Fk

\section{INTRODUCTION}

The study of superconductivity in proximity with ferromagnetic materials has opened the path towards creation and control of spin-polarized Cooper pairs and superconducting spin currents ${ }^{110}$ Recent developments show that also energy currents can be managed by using spin-polarized Cooper pairs 11 15. A considerable amount of work has concentrated on spin-polarized supercurrents across ferromagnetic metals or insulators. Hybrid structures in which superconductors are connected by a weak link of a normal-metal/ferromagnet bilayer or a ferromagnet/normal-metal/ferromagnet trilayer forming a bridge between the superconducting banks have been studied to a lesser extend. Theoretical proposals for such structures ${ }^{16}$ have been followed by experimental work on hybrid planar $\mathrm{Al}-(\mathrm{Cu} \mid \mathrm{Fe})-\mathrm{Al}$ submicron bridges ${ }^{17}$, and by further theoretical investigations to optimize practical performance 18

In the present work we study the case of a weak link consisting of a superconductor/ferromagnetic-metal bilayer, where the superconducting material is the same as in the leads, and where superconductivity is suppressed due to proximity coupling to the ferromagnetic metal, e.g. as in an $\mathrm{Al}-(\mathrm{Al} \mid \mathrm{Co})-\mathrm{Al}$ structure. A schematic illustration of the system is depicted in Fig.1. an experimental realization could be a superconducting strip running across a ferromagnetic disc. In our modeling, the structure consists in total of two blocks; the superconductor and the ferromagnet which are connected by an interface over a length $d_{f}$ (dashed line in Fig. 1), building the weak link. A superconductor in proximity with a ferromagnet exhibits spin-polarized Cooper pairs, which can be considered as a mixture between spin-singlet and spin-triplet pairs. This in turn implies a spin-polarized excitation spectrum, resulting in a spin-magnetization of the superconductor in the region where it is proximity- coupled to the ferromagnet ${ }^{19}$ (see dashed-dotted line in Fig. 1). The singlet superconducting order parameter is shown in Fig. 1 as full line, exhibiting the suppression in the proximity-coupled region.

We employ a Green function technique for metals, itinerant ferromagnets, and superconductors in the diffusive limit. Within this theory, Green functions are described by transport equations of the kind derived by Usadel, 2 generalized to spin-dependent phenomena within a Riccati representation.223|24 The considered structure makes it necessary to self-consistently calculate the pair potential with the spectrum of excitations (as encoded by the Green functions). Due to the presence of the ferromagnet, we supplement the transport equations for the Green functions with spin-dependent

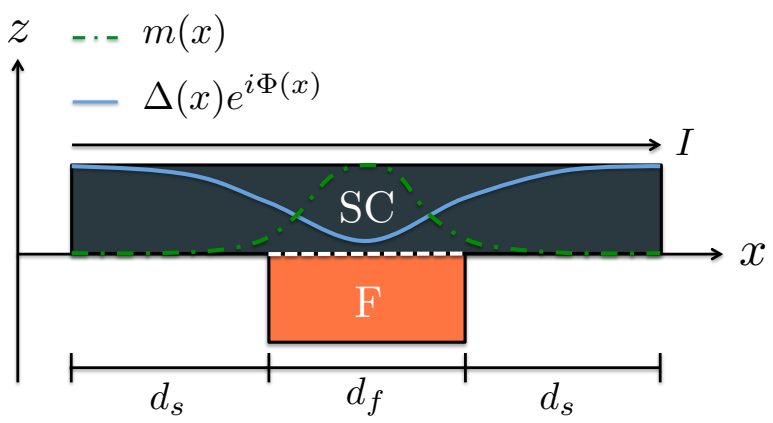

Figure 1: Model of an S-(S|F)-S junction. The dashed line between the superconductor (SC) and the ferromagnet (F) indicates a spin-active interface. The structure is of length $2 d_{s}+d_{f}$, where $d_{s}$ is the length of the superconducting banks on either side of the ferromagnet, and $d_{f}$ is the length of the superconductor/ferromagnet proximity block. The pair potential $\Delta e^{i \Phi}$ (blue, solid) and the spin-magnetization $m$ (green, dot-dashed) are shown schematically as a function of spatial coordinate $x$. A supercurrent $I$ is driven by a spatially varying superconducting phase $\Phi(x)$. 
boundary conditions. ${ }^{25}$ We propose a model in which spin-dependent interface scattering phase shifts ${ }^{20126}$ lead to a spin-polarization of Cooper pairs in the superconducting regions of the weak link. Assuming the thickness of the superconductor within the weak link much smaller than the superconducting coherence length, we are able to cast the boundary conditions in the form of an effective self-energy, which enters a one-dimensional transport equation in direction of the weak link.

In Section II we present the theoretical framework to describe our model. We supplement the Usadel equation by a self-energy-like contribution that is derived in the framework of an Andreev circuit theory to account for the spin-dependent boundary conditions.

In Section [II] we calculate characteristic observables such as the local density of states, the spin magnetization of the system, the superconducting order parameter, the characteristic current-phase relationship, and the temperature-dependence of the critical Josephson current. All calculations are performed self-consistently.

In Section IV] we explicitly show that our model fulfills the requirement of charge conservation.

In Section $\mathrm{V}$ we numerically investigate an S-F-S heterostructure that exhibits a magnetic domain wall. We extend previous work ${ }^{23}$ by a self-consistent calculation of the pair-potential, and calculate the local density of states, current-phase relations, and the temperaturedependent critical current.

\section{THEORETICAL DESCRIPTION}

We employ for our theoretical treatment Usadel theory of diffusive superconductors, ${ }^{22 \mid 27}$ adapted for spinpolarized systems (see e.g. Ref. 9). Usadel theory can be derived from the theory of Eilenberger ${ }^{28}$ and of Larkin and Ovchinnikov 29 in the diffusive limit. The equilibrium physics is captured by the retarded Green function (or propagator) $\hat{\mathbb{G}}^{R} \equiv \hat{\mathbb{G}}(\mathbf{R}, \epsilon)$ where $\mathbf{R}$ denotes the spatial coordinate, $\mathbf{R}=(x, y, z)$, and $\epsilon$ the energy. Current transport will be considered in $x$-direction, whereas $z$ denotes the direction perpendicular to the superconducting films. The propagator $\hat{\mathbb{G}}(\mathbf{R}, \epsilon)$ has a total of 16 complex-valued components and is build up of four $2 \times 2$ block spin-matrices, two of which are related to the other two by particle-hole conjugation symmetry. This matrix structure arises from the internal degrees of freedom: the spin degree of freedom and the particle-hole degree of freedom. The hat accent denotes the $2 \times 2$ block matrix structure in particle-hole (Nambu-Gor'kov) space:

$$
\hat{\mathbb{G}}=\left(\begin{array}{ll}
\mathfrak{G} & \mathfrak{F} \\
\tilde{\mathfrak{F}} & \tilde{\mathfrak{G}}
\end{array}\right)
$$

where $\mathfrak{G}, \mathfrak{F}, \tilde{\mathfrak{G}}$, and $\tilde{\mathfrak{F}}$ are $2 \times 2$ spin matrices, i.e. $\mathfrak{F}_{\alpha \beta}$ has spin indices $\alpha, \beta=\{\uparrow, \downarrow\}$ etc. The off-diagonal elements $\mathfrak{F}$ and $\tilde{\mathfrak{F}}$ quantify the superconducting pair correlations. The propagator can be analytically continued from the real energy axis into the upper complex half plane, $\epsilon \rightarrow \varepsilon$ with $\operatorname{Im}(\varepsilon) \geq 0$. The symmetry relation (particle-hole conjugation) between the block spin-matrices is given by the "tilde"-operation:

$$
\tilde{\mathfrak{A}}(\mathbf{R}, \varepsilon)=\mathfrak{A}\left(\mathbf{R},-\varepsilon^{*}\right)^{*}
$$

where $\left(^{*}\right)$ denotes complex conjugation.

In addition to the discreet internal degrees of freedom, there are continuous external degrees of freedom, which are described by the energy $\epsilon$ and the spatial coordinate $\mathbf{R}$. The diffusive motion is described by a quantum kinetic transport equation, in our case the Usadel equation, 22 which for the propagator within the superconductor, $\hat{\mathbb{G}}_{S c}(\mathbf{R}, \epsilon)$, takes the form

$$
\left[\epsilon \hat{\tau}_{3}-\hat{\Delta}, \hat{\mathbb{G}}_{S c}(\mathbf{R}, \epsilon)\right]+\frac{D}{\pi} \nabla\left(\hat{\mathbb{G}}_{S c}(\mathbf{R}, \epsilon) \nabla \hat{\mathbb{G}}_{S c}(\mathbf{R}, \epsilon)\right)=\hat{0}
$$

where $[\hat{A}, \hat{B}] \equiv \hat{A} \hat{B}-\hat{B} \hat{A}$, the $4 \times 4$ matrix $\hat{\tau}_{3}$ is the direct product between the third Pauli matrix in particle-hole space and the spin unit matrix, 0 is the $4 \times 4$ zero matrix, $\nabla \equiv \partial / \partial \mathbf{R}$, and $D$ is the diffusion constant. This transport equation is supplemented by the normalization condition

$$
\hat{\mathbb{G}}_{S c}(\mathbf{R}, \epsilon)^{2}=-\pi^{2} \hat{1},
$$

where $\hat{1} \equiv \mathbb{1}_{4 x 4}$ is the $4 \times 4$ unit matrix.

For the system depicted in Fig. 11 we make a simplifying ansatz that allows us to transform the Usadel equation into a quasi-one dimensional differential equation, supplemented by a self-energy-like contribution that accounts for the influence of the ferromagnet on the superconductor. This ansatz is motivated by assuming that the superconductor of thickness $d$ does not extend significantly in the $z$ direction, meaning that the spatial variations of the superconducting order parameter in the $z$-direction are small. This is justified for example for a superconducting strip whose lateral dimensions are much bigger than its vertical extension. Our perturbative ansatz for the Green function of the system depicted in Fig. 11 is thus:

$$
\hat{\mathbb{G}}_{S c}(x, z, \epsilon)=\hat{\mathbb{G}}_{0}(x, \epsilon)+\hat{\mathbb{G}}_{1}(x, \epsilon)(z-d)^{2}
$$

with the normalization condition

$$
\begin{aligned}
\hat{\mathbb{G}}_{S c}(x, z, \epsilon)^{2} & =\left(\hat{\mathbb{G}}_{0}(x, \epsilon)+\hat{\mathbb{G}}_{1}(x, \epsilon)(z-d)^{2}\right)^{2} \\
& =-\pi^{2} \hat{1} \quad \forall(x, z, \epsilon) .
\end{aligned}
$$

Up to linear order in $(z-d)$ this means

$$
\hat{\mathbb{G}}_{0}(x, \epsilon)^{2}=-\pi^{2} \hat{1} \quad \forall(x, \epsilon) .
$$

The surfaces at $z=d$ border to an insulating $(I)$ region. The boundary conditions at the $S / I$ interface must satisfies Nazarov's boundary conditions ${ }^{\sqrt{22}} \partial_{z} \hat{\mathbb{G}}_{S c}(x, z=$ 
$d, \epsilon)=0$. A linear contribution of the form $(z-d)$ in Eq. (5) does not satisfy this condition and therefore the ansatz for the spatial variation in the $z$-direction contains only a quadratic contribution, proportional to $(z-d)^{2}$.

To leading order in $(z-d)$ the Usadel equation reads

$$
\begin{aligned}
{\left[\epsilon \hat{\tau}_{3}-\hat{\Delta}, \hat{\mathbb{G}}_{0}(x, \epsilon)\right] } & +\frac{D}{\pi} \partial_{x}\left[\hat{\mathbb{G}}_{0}(x, \epsilon) \partial_{x} \hat{\mathbb{G}}_{0}(x, \epsilon)\right] \\
& +2 \frac{D}{\pi} \hat{\mathbb{G}}_{0}(x, \epsilon) \hat{\mathbb{G}}_{1}(x, \epsilon)=\hat{0}
\end{aligned}
$$

The contribution $\hat{\mathbb{G}}_{1}(x, \epsilon)$ will be determined from the boundary conditions of the problem and will thus depend on the structure of the ferromagnet. A detailed derivation of this expression can be seen further below, in Eq. 42. Here we note that we will show that the Usadel Equation (8) can be cast into the form

$$
\begin{aligned}
{\left[\epsilon \hat{\tau}_{3}-\hat{\Delta}-\hat{\Sigma}(x, \epsilon), \hat{\mathbb{G}}_{0}(x, \epsilon)\right] } & \\
& +\frac{D}{\pi} \partial_{x}\left[\hat{\mathbb{G}}_{0}(x, \epsilon) \partial_{x} \hat{\mathbb{G}}_{0}(x, \epsilon)\right]=\hat{0},
\end{aligned}
$$

where $\hat{\Sigma}(x, \epsilon)$ formally appears like a self-energy contribution to the system and captures the influence of the ferromagnet. It is defined in Eq. 43 below.

\section{A. Riccati Parameterization}

The Green functions can be described in the framework of the spin-dependent Riccati parameterization. ${ }^{30}$ This parameterization allows to retain the full spin structure of the Green function while automatically ensuring the normalization condition. The power of this parameterization for diffusive systems was exemplified, for example, by calculating the effects of the superconducting proximity effect through magnetic domain walls. ${ }^{23}$ Within this framework the retarded Green function $\hat{\mathbb{G}}_{0}(x, \epsilon)$ is parameterized by

$$
\hat{\mathbb{G}}_{0}=-i \pi \hat{N}\left(\begin{array}{cc}
(1+\gamma \tilde{\gamma}) & 2 \gamma \\
-2 \tilde{\gamma} & -(1+\tilde{\gamma} \gamma)
\end{array}\right)
$$

where 1 is the spin unit matrix, and where

$$
\hat{N}=\left(\begin{array}{cc}
(1-\gamma \tilde{\gamma})^{-1} & 0 \\
0 & (1-\tilde{\gamma} \gamma)^{-1}
\end{array}\right)
$$

automatically ensures the normalization condition (7). The coherence functions $\gamma$ and $\tilde{\gamma}$ are spin matrices, $\gamma_{\alpha \beta}$ with $\alpha, \beta=\{\uparrow, \downarrow\}$, where each element depends on the energy $\epsilon$ and the spatial coordinate $x$.

We now write the transport equations Eq. (9) in the Riccati parameterization. The $4 \times 4$ matrix $\hat{\Sigma}(x, \epsilon)$ is only non-zero in the range where the proximity effect between the superconductor and the ferromagnet is in action, and can be written in $2 \times 2$ block structure

$$
\hat{\Sigma}(x, \epsilon)=\left(\begin{array}{cc}
\mathfrak{A} & \mathfrak{B} \\
\tilde{\mathfrak{B}} & \tilde{\mathfrak{A}}
\end{array}\right) .
$$

With this definition, the Usadel equations for the coherence functions $\gamma$ and $\tilde{\gamma}$ are written as 2,23

$$
\begin{aligned}
& \frac{d^{2} \gamma}{d x^{2}}+\left(\frac{d \gamma}{d x}\right) \frac{\tilde{\mathfrak{F}}}{i \pi}\left(\frac{d \gamma}{d x}\right)= \\
& \frac{i}{D}\left[\gamma\left(\Delta^{*}+\tilde{\mathfrak{B}}\right) \gamma-(\epsilon 1-\mathfrak{A}) \gamma-\gamma(\epsilon 1+\tilde{\mathfrak{A}})-\Delta-\mathfrak{B}\right], \\
& \frac{d^{2} \tilde{\gamma}}{d x^{2}}+\left(\frac{d \tilde{\gamma}}{d x}\right) \frac{\mathfrak{F}}{-i \pi}\left(\frac{d \tilde{\gamma}}{d x}\right)= \\
& \frac{-i}{D}\left[\tilde{\gamma}(\Delta+\mathfrak{B}) \tilde{\gamma}+(\epsilon 1+\tilde{\mathfrak{A}}) \tilde{\gamma}+\tilde{\gamma}(\epsilon 1-\mathfrak{A})-\Delta^{*}-\tilde{\mathfrak{B}}\right]
\end{aligned}
$$

with

$$
\hat{\tau}_{3}=\left(\begin{array}{cc}
1 & 0 \\
0 & -1
\end{array}\right), \quad \hat{\sigma}_{i}=\left(\begin{array}{cc}
\sigma_{i} & 0 \\
0 & \sigma_{i}^{*}
\end{array}\right), \quad \hat{\Delta}=\left(\begin{array}{cc}
0 & \Delta \\
\Delta^{*} & 0
\end{array}\right)
$$

where $\sigma_{i}$ are the Pauli spin-matrices with $i=x, y, z$. The (temperature-dependent) spin-singlet superconducting order-parameter is given by

$$
\Delta(x)=\Delta(x) i \sigma_{y}=\Delta_{0}(x) e^{i \Phi(x)} \cdot i \sigma_{y}
$$

where $\Delta_{0}(x)$ is the modulus of the order parameter, and $\Phi(x)$ denotes a spatially dependent, real phase. The order parameter $\Delta(x)=\Delta_{0}(x) e^{i \Phi(x)}$ must be determined self-consistently as described further below, to ensure current conservation across the weak link. The Usadel equation must be supplemented by appropriate boundary conditions. This will be addressed in the next section.

\section{B. Andreev circuit theory}

We wish to employ spin-dependent boundary conditions to couple the ferromagnet to the superconductor. A crucial quantity at a boundary between a strongly spin-polarized ferromagnet and a superconductor is the spin-mixing parameter 20 , or spin-mixing conductance $\mathcal{G}_{\phi} 11,25.26 .31$. This parameter is the crucial quantity leading to spin-polarization of Cooper pairs as well as to a spin-split local density of states at the contact, and results from spin-dependent scattering phase shifts during reflection and transmission at a superconductorferromagnet interface. 20.26.31

In order to implement boundary conditions, we utilize a discretized (Andreev) quantum circuit theory $\underline{32,33}$ where the system consists of terminals, nodes, and connectors, as depicted in Figs. 2 and 3 . Within the proximity region, at each spatial point $x$ the superconductor is tunnel-coupled to a central node $C$. This coupling is characterized by a boundary conductance $\mathcal{G}_{S}$. The node itself is in contact to a ferromagnetic metal via a spin-dependent coupling that is characterized by its polarization $\mathcal{P}$, its boundary conductance value $\mathcal{G}$, and the spin-mixing parameter $\mathcal{G}_{\phi}$. 


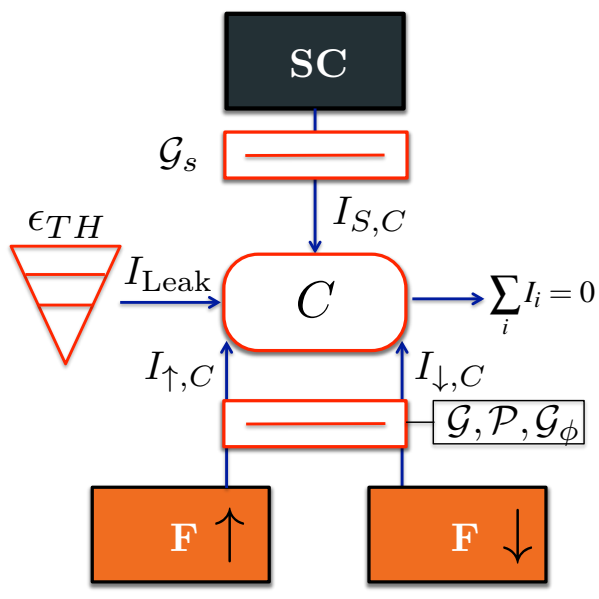

Figure 2: Illustration of an Andreev circuit to calculate the Green function $\hat{\mathbb{G}}_{C}$ of the central node $C$. It consists of a central node $C$ that is connected to a ferromagnetic terminal by a connector that is characterized by a set of conductance parameters $\mathcal{G}, \mathcal{P}, \mathcal{G}_{\phi}$ (see text). The leakage terminal is characterized by a Thouless energy $\epsilon_{\mathrm{Th}}$. The arrows between the blocks indicate the flow of matrix currents that obey a Kirchhoff rule, see Eq. (17).

The loss of superconducting correlations is accounted for by a leakage current that contains the Thouless energy $\epsilon_{\mathrm{Th}}$ of the leakage terminal. The central node $C$ is responsible to model the behavior of the superconducting correlations in the structure under the effect of leakages and spin-polarized boundaries of the ferromagnet. As has been shown by Nazarov $\sqrt{32}$, the following generalized Kirchhoff rule for the so-called matrix current holds (see Fig. 2):

$$
\hat{I}_{S, C}+\hat{I}_{\downarrow, C}+\hat{I}_{\uparrow, C}+\hat{I}_{\text {Leak }}=\hat{0}
$$

where $\hat{I}_{S, C}$ is the matrix current from the superconductor to the node $C$. The matrix currents from the ferromagnet into the central node $C$ are denoted by $\hat{I}_{\downarrow, C}, \hat{I}_{\uparrow, C}$ whereas $\hat{I}_{\text {Leak }}$ is the matrix current from the leakage terminal going into the central node. Eq. (17) has to be applied at each interface point $(x, z)$ with $z=0$ at the interface between the superconductor and the ferromagnet (see Fig. 3).

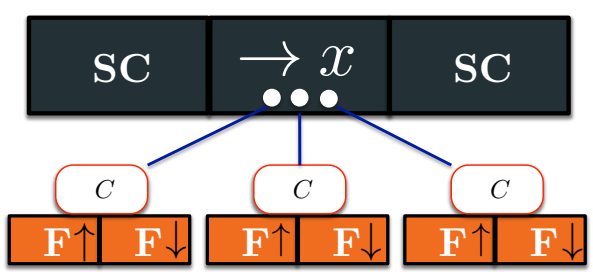

Figure 3: The Green function $\hat{\mathbb{G}}_{C}$ of the central node has to be calculated for every point $x$ at the interface and for all energies. The red blocks labeled $C$ and $F$ refer to the central node $C$ and the ferromagnet blocks discussed in Fig.2
The leakage current is given by

$$
\hat{I}_{\text {Leak }}(x, \epsilon)=\frac{\mathcal{G}_{q}}{4 \epsilon_{\mathrm{Th}}}\left[\hat{\mathbb{G}}_{\text {Leak }}(\epsilon), \hat{\mathbb{G}}_{C}(x, \epsilon)\right]
$$

where $\hat{\mathbb{G}}_{\text {Leak }}(\epsilon)=-\pi \epsilon \hat{\tau}_{3}$ is an energy-dependent quantity to account for a leakage of coherence. All information about the leakage terminal is given by its Thouless energy $\epsilon_{\mathrm{Th}}$.

The matrix current between the terminals $j$ and the central node $C$ in linear order in $\mathcal{T}_{n}$ (see below) can be written in the form of the following commutator ${ }^{11113}$

$$
\begin{aligned}
\hat{I}_{j, C}=\frac{1}{2}\left[\mathcal{G}_{0, j} \cdot \hat{\mathbb{G}}_{j}+\mathcal{G}_{P, j} \cdot\left\{\hat{\kappa}_{j}, \hat{\mathbb{G}}_{j}\right\}\right. & \\
& \left.\quad+\mathcal{G}_{1, j} \cdot \hat{\kappa}_{j} \hat{\mathbb{G}}_{j} \hat{\kappa}_{j}-\pi \mathcal{G}_{\phi, j} \cdot \hat{\kappa}_{j}, \hat{\mathbb{G}}_{C}\right]
\end{aligned}
$$

where $j \in\{\uparrow, \downarrow, S\}$ labels the terminal. The boundary conditions are specified by the set of conductance parameters 11,13

$$
\begin{aligned}
& \mathcal{G}_{0}=\mathcal{G}_{q} \sum_{n}^{\text {\#Channels }} \mathcal{T}_{n}\left(1+\sqrt{1-\mathcal{P}_{n}^{2}}\right) \\
& \mathcal{G}_{1}=\mathcal{G}_{q} \sum_{n}^{\text {\#Channels }} \mathcal{T}_{n}\left(1-\sqrt{1-\mathcal{P}_{n}^{2}}\right) \\
& \mathcal{G}_{P}=\mathcal{G}_{q} \sum_{n}^{\# \text { Channels }} \mathcal{T}_{n} \mathcal{P}_{n} \\
& \mathcal{G}_{\phi}=2 \mathcal{G}_{q} \sum_{n}^{\# \text { Channels }} \delta \Phi_{n} .
\end{aligned}
$$

Here, the spin-mixing parameter is described by $\delta \Phi_{n}$, the spin polarization of the ferromagnet by $\mathcal{P}_{n}$, the spinaveraged transmission probability for channel $n$ is given by $\mathcal{T}_{n}$ and $\mathcal{G}_{q}=\frac{e^{2}}{h}$ is the quantum conductance.

For a strongly spin-polarized ferromagnet the transmission and reflection channels at the interface are completely spin-polarized $\left(\mathcal{P}_{n}=1\right.$ and -1 for spin-up and spin-down, respectively), such that we obtain

$$
\begin{aligned}
& \mathcal{G}_{0}=\mathcal{G}_{1}=\mathcal{G}_{q}\left(\sum_{n}^{\# \uparrow} \mathcal{T}_{n}+\sum_{n}^{\# \downarrow} \mathcal{T}_{n}\right) \equiv \frac{1}{2}\left(\mathcal{G}_{\uparrow}+\mathcal{G}_{\downarrow}\right) \\
& \mathcal{G}_{P}=\mathcal{G}_{q}\left(\sum_{n}^{\# \uparrow} \mathcal{T}_{n}-\sum_{n}^{\# \downarrow} \mathcal{T}_{n}\right) \equiv \frac{1}{2}\left(\mathcal{G}_{\uparrow}-\mathcal{G}_{\downarrow}\right) \\
& \mathcal{G}_{\phi}=2 \mathcal{G}_{q}\left(\sum_{n}^{\# \uparrow} \delta \Phi_{n}-\sum_{n}^{\# \downarrow} \delta \Phi_{n}\right) \equiv \mathcal{G}_{\phi, \uparrow}-\mathcal{G}_{\phi, \downarrow}
\end{aligned}
$$

where $\# \uparrow$ is the number of spin-up channels and \# $\downarrow$ the number of spin-down channels.

We obtain the matrix current between the superconductor and the central node $C$ by setting $\mathcal{G}_{1, S}=\mathcal{G}_{P, S}=$ $\mathcal{G}_{\phi, S}=0$ and defining $\mathcal{G}_{0, S}=\mathcal{G}_{S}$ for the superconductor. The expression for the ferromagnetic contacts 
is simplified by $\left[\hat{\mathbb{G}}_{j}, \hat{\kappa}_{j}\right]=0$; thus, one can combine $\mathcal{G}_{\uparrow,(\downarrow)}=\mathcal{G}_{0, \uparrow(\downarrow)}+\mathcal{G}_{1, \uparrow(\downarrow)}$. Furthermore, we simplify the notation by setting $\hat{I}_{j, C} \equiv \hat{I}_{j}$. The various matrix currents are then determined by the following boundary conditions 9911 .

$$
\begin{aligned}
\hat{I}_{\alpha}(x, \epsilon)= & \frac{1}{2}\left[\mathcal{G}_{\alpha} \cdot \hat{\mathbb{G}}_{\alpha}^{F}+\mathcal{G}_{P, \alpha} \cdot\left\{\hat{\kappa}_{\alpha}, \hat{\mathbb{G}}_{\alpha}^{F}\right\}\right. \\
& \left.-\pi \mathcal{G}_{\phi, \alpha} \cdot \hat{\kappa}_{\alpha}, \hat{\mathbb{G}}_{C}(x, \epsilon)\right] \\
\hat{I}_{S}(x, \epsilon)= & \frac{1}{2} \mathcal{G}_{S} \cdot\left[\hat{\mathbb{G}}_{0}(x, \epsilon), \hat{\mathbb{G}}_{C}(x, \epsilon)\right]
\end{aligned}
$$

where $\alpha \in\{\uparrow, \downarrow\}$, and

$$
\begin{aligned}
\hat{\kappa}_{\uparrow} & =-\hat{\kappa}_{\downarrow} \equiv \hat{\kappa}=\mathbb{1}_{2 \times 2} \otimes(\mathbf{m} \cdot \boldsymbol{\sigma}), \\
\hat{\mathbb{G}}_{\uparrow}^{F} & =\hat{\mathbb{G}}_{\downarrow}^{F}=-i \pi \hat{\tau}_{3} \equiv \hat{\mathbb{G}}^{F} .
\end{aligned}
$$

Here, $\hat{\mathbb{G}}^{F}$ is the solution to the Usadel equation (ß) for a non-superconducting material $(\hat{\Delta}=\hat{0})$. The direction of the magnetization of the ferromagnet is described by the spin-matrix $\hat{\kappa}$, where $\boldsymbol{m}$ is the unit vector of magnetization of the interface and $\boldsymbol{\sigma}$ is the vector of spin Pauli matrices. $\hat{\tau}_{0}$ is the unit matrix in $2 \times 2 \mathrm{Nambu-Gor'kov}$ space. The Green function $\hat{\mathbb{G}}_{0}(x, \epsilon)$ is the Green function defined in Eq. (10) that solves Eq. (13) and Eq. (14).

More compactly, we can write:

$$
\begin{aligned}
\hat{I}_{F}(x, \epsilon) & =\hat{I}_{\downarrow}+\hat{I}_{\uparrow} \\
= & \frac{1}{2}\left[\mathcal{G} \hat{\mathbb{G}}^{F}+\mathcal{G}_{P}\left\{\hat{\kappa}, \hat{\mathbb{G}}^{F}\right\}-\pi \mathcal{G}_{\phi} \hat{\kappa}, \hat{\mathbb{G}}_{C}(x, \epsilon)\right],
\end{aligned}
$$

and the boundary of the ferromagnet to the superconductor is characterized by the three parameters $\mathcal{P}, \mathcal{G}$, and $\mathcal{G}_{\phi}$, see Fig. \&,

$$
\begin{aligned}
\mathcal{G} & =\mathcal{G}_{\uparrow}+\mathcal{G}_{\downarrow}, \\
\mathcal{G}_{P} & =\mathcal{G}_{P, \uparrow}+\mathcal{G}_{P, \downarrow}=\frac{1}{2}\left(\mathcal{G}_{\uparrow}-\mathcal{G}_{\downarrow}\right)=\frac{1}{2} \mathcal{G} \mathcal{P}, \\
\mathcal{P} & =\frac{\mathcal{G}_{\uparrow}-\mathcal{G}_{\downarrow}}{\mathcal{G}_{\uparrow}+\mathcal{G}_{\downarrow}}, \\
\mathcal{G}_{\phi} & =\mathcal{G}_{\phi, \uparrow}-\mathcal{G}_{\phi, \downarrow} .
\end{aligned}
$$

Here, $\mathcal{G}_{P}$ and $\mathcal{G}$ refer to conductances given in terms of spin-dependent boundary conductances $\mathcal{G}_{\uparrow, \downarrow}$. A spinpolarized boundary necessarily leads to spin-dependent scattering phases that are accounted for by a parameter $\mathcal{G}_{\phi}$ which is the most relevant parameter to modify the superconducting correlations. This modification appears in the pair amplitudes $(\mathfrak{F}, \tilde{\mathfrak{F}})$ of the structure. This can be thought of as the ferromagnet imprinting its magnetic correlations to the proximity coupled superconductor in its immediate vicinity which influences the transport properties of the structure.

\section{Determination of the Green function $\hat{\mathbb{G}}_{C}$ of the central node}

The Green function $\hat{\mathbb{G}}_{C}$ is calculated within Andreev circuit theory and is used to evaluate the ferromag- netic influence on the transport properties of the system through the superconductor via a self-energy contribution to the Usadel equation. From the Kirchhoff rule, Eq. (7), the contact Green Function $\hat{\mathbb{G}}_{C}$ in the central node $C$ is determined by solution of the equation

$$
\left[\hat{M}(x, \epsilon), \hat{\mathbb{G}}_{C}(x, \epsilon)\right]=\hat{0}
$$

where $\hat{M}(x, \epsilon)$ is given by

$$
\begin{gathered}
\hat{M}(x, \epsilon)=\frac{\mathcal{G}_{q}}{4 \epsilon_{\mathrm{Th}}} \hat{\mathbb{G}}_{\text {Leak }}(\epsilon)+\frac{1}{2} \mathcal{G} \cdot \hat{\mathbb{G}}^{F}+\frac{1}{2} \mathcal{G}_{P} \cdot\left\{\hat{\kappa}, \hat{\mathbb{G}}^{F}\right\} \\
-\frac{\pi}{2} \mathcal{G}_{\phi} \cdot \hat{\kappa}+\frac{1}{2} \mathcal{G}_{S} \cdot \hat{\mathbb{G}}_{0}(x, \epsilon) .
\end{gathered}
$$

Eq. (36) is supplemented by the normalization condition

$$
\hat{\mathbb{G}}_{C}^{2}=-\pi^{2} \hat{1},
$$

which means that (a) the matrix $\hat{\mathbb{G}}_{C}$ is diagonalizable and (b) the only eigenvalues of $\hat{\mathbb{G}}_{C}$ are $\pm i \pi$. Eq. (36) then ensures that if $\hat{M}$ is diagonalizable (which in our case holds true), then $\hat{M}$ and $\hat{\mathbb{G}}_{C}$ can be diagonalized simultaneously and have a common set of eigenvectors. Additionally, we demand that the eigenvalues of the contact Green function be continuously connected to those of the normal state. 13.32 With these constraints the Green function $\hat{\mathbb{G}}_{C}$ is written as

$$
\hat{\mathbb{G}}_{C}(x, \epsilon)=i \pi \hat{U}_{M}^{-1} \operatorname{sgn}\left[\operatorname{Im}\left(\hat{D}_{M}\right)\right] \hat{U}_{M}
$$

with $\hat{D}_{M}, \hat{U}_{M}$ containing the eigenvalues and eigenvectors of matrix $\hat{M}$, respectively, and sgn denoting the sign function applied to the imaginary part of each eigenvalue.

One can now calculate measurable quantities such as the density of states which depends on the set of parameters $\mathcal{G}, \mathcal{P}, \mathcal{G}_{\phi}$. The parameter $\mathcal{G}_{\phi}$ has a similar effect on the density of states as a ferromagnetic exchange field. A non-zero value of $\mathcal{G}_{\phi}$ spin-splits the density of states in the central node $C$, see Fig. 4. On the left in this figure we plot as an example the density of states inside the central node $C$ by taking the analytic value for a homogeneous superconductor $\widehat{G}_{0}(\epsilon)$ (independent of spatial coordinate $x$ ). This result agrees with the one shown in Ref. 11. On the right hand side in Fig. 4 we show a typical example for the density of states inside the central node $C$ at coordinate $x=6 \xi$ for the S- $(\mathrm{F} \mid \mathrm{S})-\mathrm{S}$ system we consider, for parameters shown in Table I $\square$

\section{Implementation of boundary conditions}

In $z$-direction we have two interfaces, an $S / F$ boundary at $z=0$ and an $S / I$ boundary at $z=d$ (see Fig. 1), where

\begin{tabular}{c|c|c|c|c|c|c}
$\mathcal{G}$ & $\rho_{S} D / A d$ & $\mathcal{G}_{\phi}$ & $\mathcal{P}$ & $\epsilon_{\mathrm{Th}} / \mathcal{G}_{q}$ & $d_{s}$ & $d_{f}$ \\
\hline $0.1 \mathcal{G}_{S}$ & $0.75 \pi$ & $0.25 \mathcal{G}_{S}$ & 0.9 & $0.51 \Delta_{0} / \mathcal{G}_{S}$ & $5.0 \xi$ & $2.0 \xi$
\end{tabular}

Table I: If not explicitly stated otherwise, we calculate all observables of our system for these parameters. 

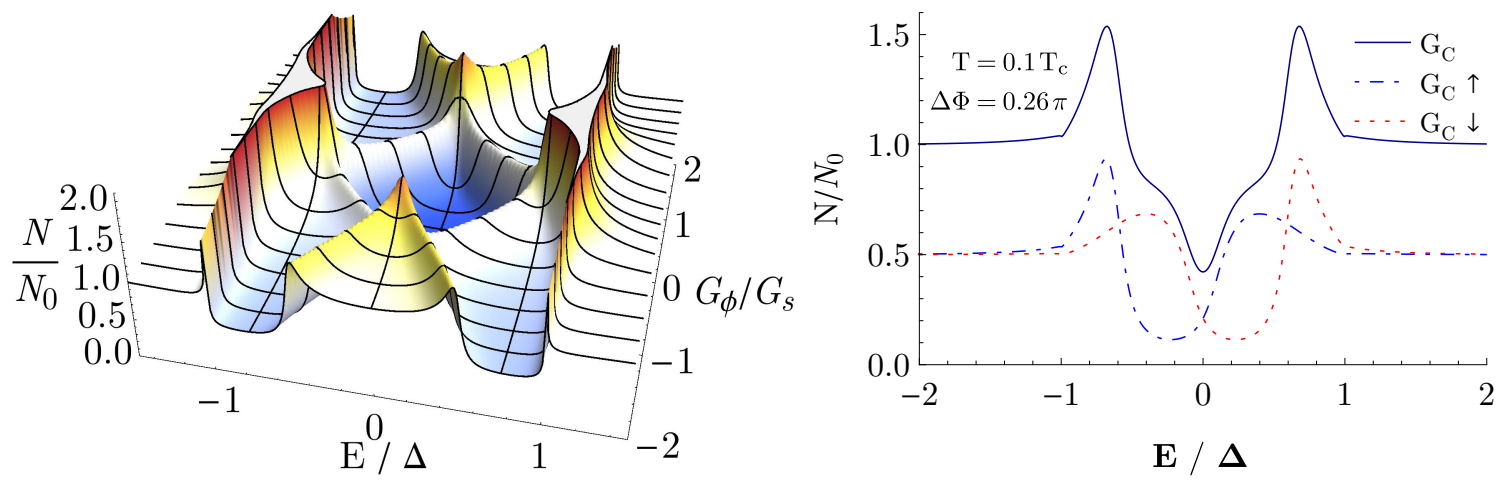

Figure 4: Density of states (DOS) $N(E)$ of the contact Green function $\hat{\mathbb{G}}_{C}$ in the central node C (see Fig. 2). $N_{0}$ is the density of states in the normal state at the Fermi energy. Left: Dependence on $\mathcal{G}_{\phi}$ and on $E / \Delta_{0}$ calculated for a homogeneous superconductor for $\Delta \Phi=0$. Right: $N / N_{0}$ at $x=6 \xi$ in the middle of the weak-link S-(S|F)-S structure for $\Delta \Phi=0.26 \pi$. In addition, the spin-resolved DOS is shown, to illustrate the spin-splitting due to a non-zero $\mathcal{G}_{\phi}$. Parameters are as in Table $\mathrm{I}$.

the Green function is subjected to boundary conditions. We use Nazarov's boundary condition for spin-active $\mathrm{e}^{1126}$ and spin-inactive interfaces ${ }^{32 \mid 33}$ to define the matrix current $\hat{I}_{S}$ in the superconductor in the vicinity of the $S / F$ and the $S / I$ interface:

$$
\hat{I}_{S}=\left.\mp \frac{A}{\rho_{S}} \hat{\mathbb{G}}_{S}(x, z, \epsilon) \frac{\mathrm{d}}{\mathrm{d} z} \hat{\mathbb{G}}_{S}(x, z, \epsilon)\right|_{z=0,(d)}
$$

where $A$ is the contact area of the boundary and the parameter $\rho_{S}$ denotes the resistivity of the $S$ material and the minus (plus) sign refers to $z=0(d)$. At the $S / I$ boundary the matrix current $I_{S}$ has to vanish, which in linear order is automatically ensured by the chosen parameterization,

$$
\hat{I}_{S}=\left.\hat{\mathbb{G}}_{0}(x, \epsilon) 2 \hat{\mathbb{G}}_{1}(x, \epsilon)(z-d)\right|_{z=d}=\hat{0} .
$$

At the $S / F$ boundary at $z=0$, the matrix current in linear order in $(z-d)$ is

$$
\begin{aligned}
\hat{I}_{S} & =-\left.\frac{A}{\rho_{S}} \hat{\mathbb{G}}_{0}(x, z, \epsilon) 2 \hat{\mathbb{G}}_{1}(x, \epsilon)(z-d)\right|_{z=0} \\
& =\frac{A \cdot d}{\rho_{S}} 2 \hat{\mathbb{G}}_{0}(x, \epsilon) \hat{\mathbb{G}}_{1}(x, \epsilon) .
\end{aligned}
$$

Matrix current conservation ${ }^{32.33}$ requires this expression to be equal to the one in Eq. 28, which leads to

$$
\hat{\mathbb{G}}_{0}(x, \epsilon) \hat{\mathbb{G}}_{1}(x, \epsilon)=-\frac{\mathcal{G}_{S} \rho_{S}}{4 A \cdot d}\left[\hat{\mathbb{G}}_{C}(x, \epsilon), \hat{\mathbb{G}}_{0}(x, \epsilon)\right] .
$$

Since the contact Green function $\hat{\mathbb{G}}_{C}(x, \epsilon)$ is known from the Kirchhoff rule Eq. (36) and $\hat{\mathbb{G}}_{0}(x, \epsilon)$ is known from the solution of the Usadel equation, this equation determines the perturbation that is defined in Eq. (9) and Eq. (8), as

$$
\hat{\Sigma}(x, \epsilon) \equiv \frac{1}{2 \pi} \frac{\rho_{S} D}{A \cdot d} \mathcal{G}_{S} \cdot \hat{\mathbb{G}}_{C}(x, \epsilon) .
$$

We summarize the iterative procedure to selfconsistently calculate the pair potential $\Delta$ and the Green function $\hat{\mathbb{G}}_{C}$ below:

1. Numerically solve the Usadel equation Eq. (9) to obtain $\hat{\mathbb{G}}_{0}(x, \epsilon) \quad \forall(x, \epsilon)$

2. Application of the Kirchhoff rules $\sum_{i} I_{i}=0$ leads to the Green function $\hat{\mathbb{G}}_{C}(x, \epsilon)$.

3. Application of spin-conserving and spin-dependent boundary conditions show that the Green function $\hat{\mathbb{G}}_{C}$ determines a self-energy contribution to the Usadel equation written as $\Sigma(x, \epsilon)$

4. Solve the Usadel equation with the new self-energy contribution $\hat{\Sigma}(x, \epsilon)$

5. Calculate the order-parameter $\Delta(x)=\Delta_{0}(x) e^{i \Phi(x)}$. $i \sigma_{y}$ by solving the mean-field self-consistency equation Eq. (48)

6. Repeat the iteration procedure until the orderparameter satisfies a convergence criterion.

Note that in the iteration cycle the self-energy $\hat{\Sigma}(x, \epsilon)$ as well as the order-parameter $\Delta(x, T)$ vary simultaneously. The self-consistent iteration cycle is repeated until both of them have converged.

\section{OBSERVABLES}

If not explicitly stated otherwise, we calculate all observables of our system for the parameters that are given in Table I. We apply a finite phase difference $\Delta \Phi \equiv$ $\Phi(x=L)-\Phi(x=0)$ to the outer superconducting electrodes, which gives rise to a Josephson current through 

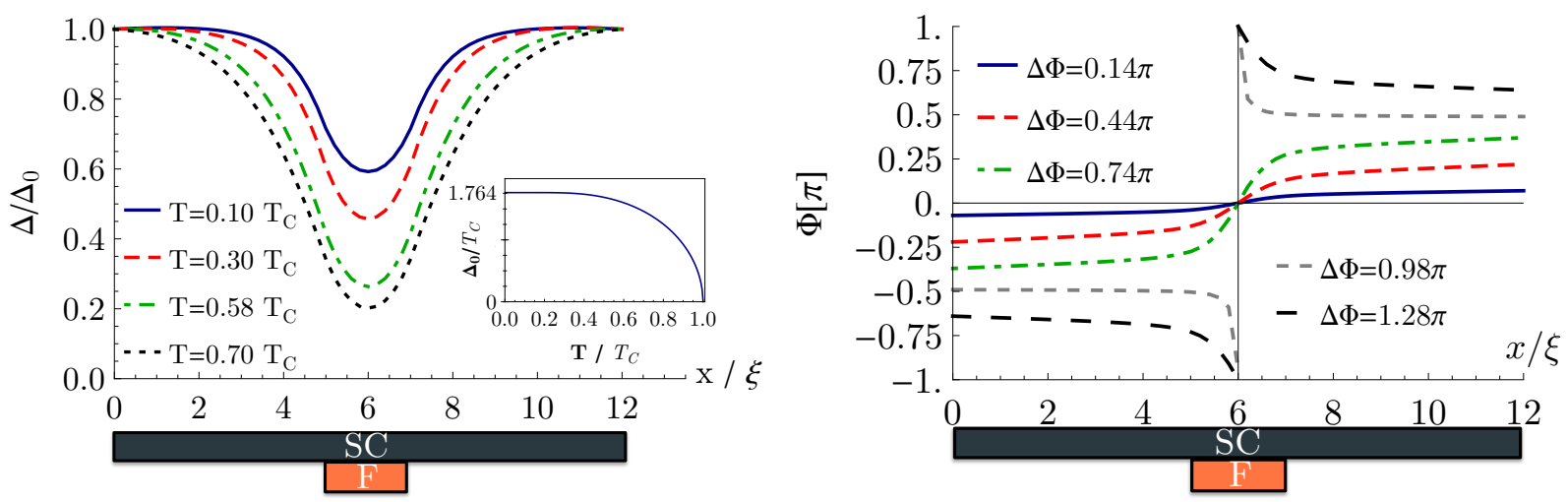

Figure 5: Left: Self-consistently calculated pair potential with Eq. 48 for various temperatures with small external phase difference $\Delta \Phi=0.26 \pi$. Each Plot has been normalized to the homogeneous value $\Delta_{0}(T)$ such that all curves are normalized. The pair potential is suppressed in the ferromagnetic region that is located at $x \in(5,7) \xi$. Inset: Temperature-dependence of the pair potential for a homogeneous BCS-superconductor. Right: Phase evolution across the system for different applied phase differences at a temperature of $T=0.58 T_{C}$

the weak link. Formally, this is introduced by the substitution:

$$
\begin{aligned}
& \Delta(x, T) \rightarrow \Delta_{0}(x, T) e^{i \Phi(x)}, \quad \Phi(x) \in \mathbb{R}, \\
& \Phi(x=\text { Left border })=-\frac{\Delta \Phi}{2}, \\
& \Phi(x=\text { Right border })=\frac{\Delta \Phi}{2} .
\end{aligned}
$$

\section{A. Pair potential and phase evolution}

The pair potential $\Delta(x, T)$ is calculated by solving a self-consistency equation. Here, we are concerned with the following $4 \times 4$ matrix structure arising from particlehole and spin degrees of freedom:

$$
\hat{\Delta}(x, T)=\left(\begin{array}{cc}
0 & \Delta(x, T) \\
\Delta^{*}(x, T) & 0
\end{array}\right)
$$

where $\Delta(x, T)=\Delta(x, T) i \sigma_{y}$.

The self-consistency equation reads

$$
\Delta(x, T)=\lim _{\epsilon_{c} \rightarrow \infty} \frac{\frac{1}{2 \pi i} \int_{-\epsilon_{c}}^{\epsilon_{c}} d \epsilon \tanh (\epsilon / 2 T) \mathfrak{F}(x, \epsilon, T)}{\int_{-\epsilon_{c}}^{\epsilon_{c}} \frac{d \epsilon}{2 \epsilon} \tanh (\epsilon / 2 T)+\ln \left(T / T_{c}\right)} .
$$

The equation is numerically evaluated with a sufficiently large, temperature-independent, energy cut-off $\epsilon_{c}$. Apart from the usual suppression of superconductivity with increasing temperature, the pair potential is strongly suppressed in the region of the ferromagnet, see on the left hand side of Fig. 5. When applying a fixed phase difference $\Delta \Phi$ to the outer superconducting electrodes, the spatial evolution of the phase is determined by the selfconsistency equation Eq. 48). The self-consistent evolution of the phase across the system is shown on the right hand side of Fig. 5. In the numerical iteration process, we fix the phase difference as well as the absolute value of the pair potential at the left and the right-hand side of our structure. The latter is given by the well-known temperature-dependence of a homogeneous BCS-type superconductor (see inset, Fig.5)

\section{B. (Local) Density of states}

The local density of states for the system is given by

$$
N(x, \epsilon)=-\frac{N_{0}}{2 \pi} \operatorname{Im}\left(\operatorname{Tr}_{2}[\mathfrak{G}(x, \epsilon)]\right) .
$$

In Fig. 6 the spatial variation of the local density of states is shown. It can be seen that the DOS retains its characteristic structure far from the $(\mathrm{S} \mid \mathrm{F})$ weak link. In the $(\mathrm{S} \mid \mathrm{F})$ weak-link region additional subgap Andreev bound

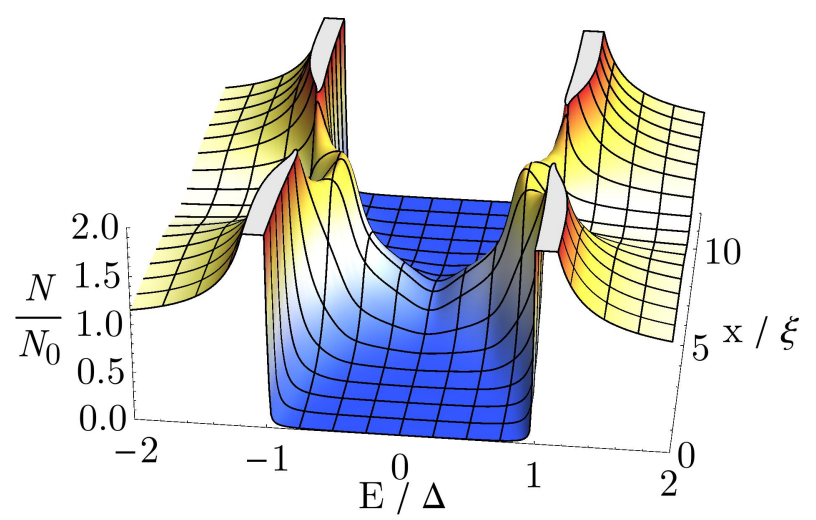

Figure 6: Density of states as a function of energy $E$ and spatial coordinate $x$. The $(\mathrm{S} \mid \mathrm{F})$ weak link extends from $x=5 \xi$ to $7 \xi$. The DOS is calculated for $\Delta \Phi=0.26 \pi$, at $T=0.1 T_{C}$. 


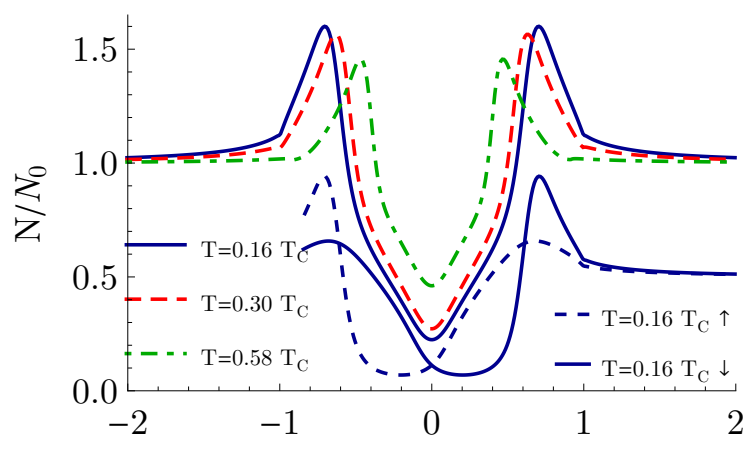

$\mathrm{E} / \Delta$

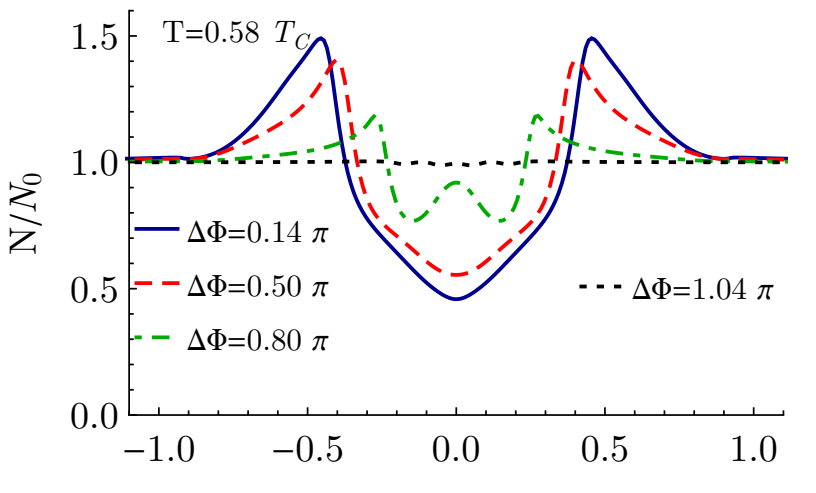

$\mathrm{E} / \Delta$

Figure 7: Left: Local density of states at $x=6 \xi$ in the weak-link structure. The LDOS is spin-split into a spin-up and a spin-down contribution due to a non-zero $\mathcal{G}_{\phi}$ component. The lines corresponding to temperatures $T_{C, \uparrow(\downarrow)}$ refer to the local spin-density of states $N_{\uparrow} / N_{0}, N_{\downarrow} / N_{0}$, respectively, with the total density of states defined as $N=N_{\uparrow}+N_{\downarrow}$ and $N_{0}$ as the density of states at the Fermi energy in the normal state. Right: Variation of the density of states at $x=6 \xi$ in the middle of the structure with an applied phase-difference $\Delta \Phi$.

states appear. These are present also in the superconducting electrodes within a coherence length from the weak-link region, in particular below the gap edges of the bulk density of states.

The local density of states at $x=6 \xi$ (the middle of the structure) is shown in Fig. 7. In the middle of the investigated structure, the local density of states for the system shows a proximity induced narrowing in its spectrum that is reminiscent of proximity induced minigaps in S-N-S systems. This narrowing is induced by a spinsplit DOS as can be seen in Fig.77. The presence of the ferromagnet, which is encoded in a non-vanishing spinmixing parameter $\mathcal{G}_{\phi}$ shifts spin-up and spin-down contributions to the density of states energetically apart. An additionally applied phase difference to the outer superconducting electrodes leads to a gradual reduction of the gap size. This is due to additional subgap Andreev bound states ${ }^{35}$, which are shifted in the presence of a superflow, see Fig.7 right. A zero-bias peak appears for sufficiently large phase differences.

\section{Spin-Magnetization}

We make use of the usual notation to express the normal and anomalous pair amplitudes $\mathfrak{G}$ and $\mathfrak{F}$, respectively,

$$
\begin{aligned}
\mathfrak{G} & =G_{0} 1+\boldsymbol{G} \cdot \boldsymbol{\sigma} \\
\mathfrak{F} & =\left(F_{0} 1+\boldsymbol{F} \cdot \boldsymbol{\sigma}\right) i \sigma_{y} .
\end{aligned}
$$

A general feature of SF-proximity influenced systems is that the presence of non-zero triplet amplitudes $\boldsymbol{F}$ entails the presence of a non-zero $G{ }^{34}$ This means that through a non-zero $G_{z}$, the spin-up and spin-down contribution to the local density of state are not degenerate any more. Thus, due to proximity to the ferromagnet, the superconductor develops a spin-magnetization in the vicinity of the $\mathrm{S} \mid \mathrm{F}$ interface ${ }^{19} \mid 21$ The induced spin-magnetization $\boldsymbol{m}(x)$ can be calculated in the following way ${ }^{\sqrt{9}}$

$$
\begin{gathered}
\boldsymbol{m}(x)=2 N_{0} T \int_{-\infty}^{\infty} \frac{d \epsilon}{4 \pi i} \quad\left[\boldsymbol{G}(x, \epsilon+i \delta) \tanh \left(\frac{\epsilon+i \delta}{2 T}\right)\right. \\
\left.-(\boldsymbol{G}(x, \epsilon+i \delta))^{\dagger} \tanh \left(\frac{\epsilon-i \delta}{2 T}\right)\right]
\end{gathered}
$$

where $N_{0}$ is the density of states at the Fermi energy.

A plot of $m_{x}(x)$ can be seen in Fig.8. A non-zero spinmagnetization is induced in the superconducting material that sits on top of the ferromagnetic material, i.e. at $x \in(5,7) \xi$. Due to the inverse proximity effect, a non-zero magnetization can penetrate into the adjacent

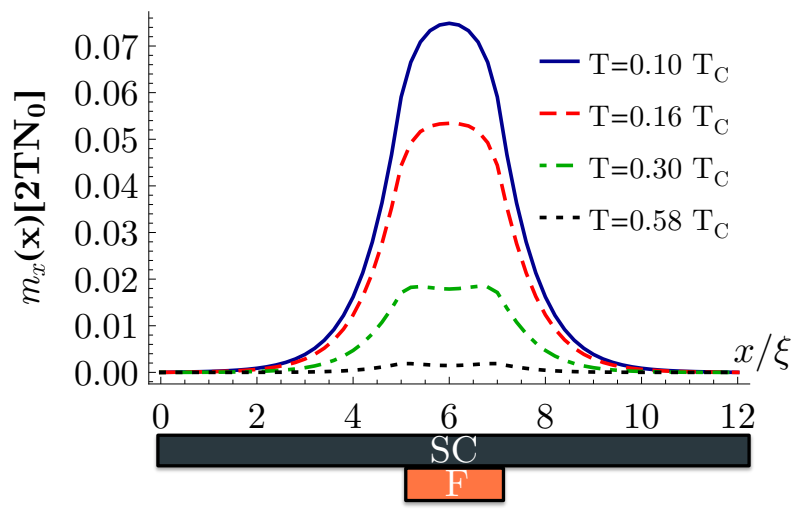

Figure 8: Induced spin-magnetization by a non-zero $\boldsymbol{G}$ component. It can be seen that the system obtains a finite spinmagnetization that is restricted to the contact region of the ferromagnet and the superconductor. Due to the inverse proximity effect and the penetration of the triplet amplitudes in the superconducting region, there is a finite magnetization for $x>7 \xi$ and for $x<5 \xi$. The applied phase-difference is $\Delta \Phi=0.26 \pi$. 
superconducting blocks as it is indicated in Fig. 8. The calculations show that the ferromagnet imprints its magnetic structure onto the superconductor.

\section{Weak-link Current-Phase Relationships}

A finite phase difference $\Delta \Phi \neq 0$ gives rise to a Josephson current through the system. The Josephson currents themselves are spatially conserved, $\partial_{x} J(x)=0$, only if the system fulfills the self-consistency equations for the pair potential, see Eq. (48). The current conservation in the presence of the self-energy correction to the Usadel Equation can be shown analytically (see section IV). The total current through the superconducting leads is denoted as $J(x)$ and is evaluated as

$$
\begin{gathered}
J=-e N_{0} \int_{-\infty}^{+\infty} \frac{d \epsilon}{2 \pi} \frac{D}{\pi} \frac{\operatorname{Tr}_{4}}{2}\left(\hat{\tau}_{3} \operatorname{Re}\left[\hat{\mathbb{G}}_{0} \partial_{x} \hat{\mathbb{G}}_{0}\right]\right) \tanh \frac{\epsilon}{2 T} \\
=\frac{\sigma_{N}}{2 e} \int_{-\infty}^{+\infty} d \epsilon \operatorname{Tr}_{2}\left[\operatorname { R e } \left(\left\{(1-\gamma \tilde{\gamma})^{-1} \partial_{x} \gamma(1-\tilde{\gamma} \gamma)^{-1}, \tilde{\gamma}\right\}_{+}\right.\right. \\
\left.\left.\quad-\left\{(1-\tilde{\gamma} \gamma)^{-1} \partial_{x} \tilde{\gamma}(1-\gamma \tilde{\gamma})^{-1}, \gamma\right\}_{+}\right)\right] \tanh \frac{\epsilon}{2 T}
\end{gathered}
$$

where $\sigma_{N}=e^{2} N_{0} D$ is the conductivity in a normal metal with diffusion coefficient $D$ and the density of states at the Fermi level $N_{0}$. Here $\{A, B\}_{+}=A B+B A$, and in the last line we have written the current in terms of the Riccati amplitudes.

The critical current is the maximum current that is realized in the system as function of phase differences $\Delta \Phi$,

$$
J_{s}(T)=\max _{\Delta \Phi}[J(\Delta \Phi, T)] .
$$

The phase-dependence for the weak link structure (S$(\mathrm{F} \mid \mathrm{S})-\mathrm{S})$ in general strongly deviates from a sinusoidal relation at low temperatures. In particular, it can become multi-valued in certain ranges of the phase difference $\Delta \Phi !^{[3}$ If the current-phase relation is single-valued, then for symmetry reasons $J(\Delta \Phi=\pi)=0$. In the case of a multi-valued current-phase relation it is however possible that the current reaches its maximum value for phases $\Delta \Phi>\pi$, before the current jumps to its negative branch at a critical value of $\Delta \Phi$.

Typical current-phase relationships are depicted in Fig. 9. At low temperatures, shortly after the current reaches its maximum value, the system can occupy two different Josephson states that only slightly differ in energy. For the numerical calculation it means that the system can oscillate between the two solutions which makes it numerically difficult to converge to a solution. Stable, converged solutions are therefore shown as symbols, whereas dotted lines show the most likely continuation for the unstable branches. For increasing temperature, the current-

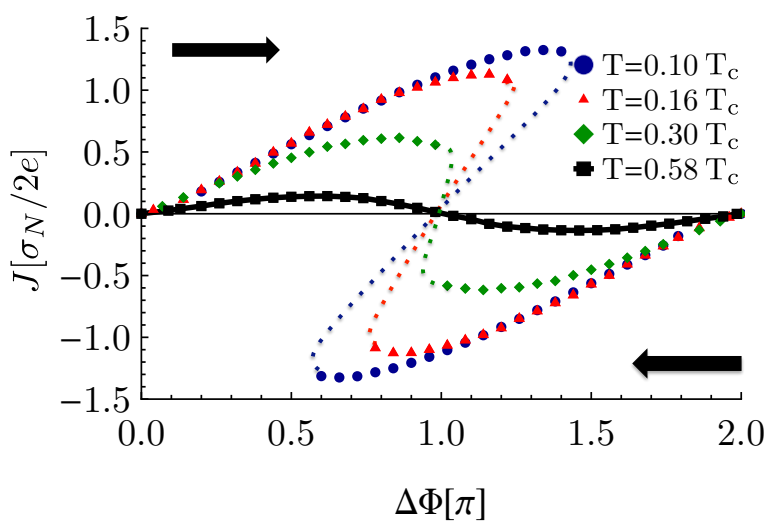

Figure 9: Crossover from a multi-valued to a sinusoidal current as the temperature is increased. As indicated by the arrows, the graphs from the upper half are calculated for an increasing phase difference, the graphs from the lower half for a decreasing phase difference. The dotted lines are schematic and indicate the most likely continuation of the current-phase relationship, as the current-phase relationship becomes unstable shortly after the maximum is reached as multiple solutions for the current become possible. We show here only the stable, converged solutions.

phase relation approaches a sinusoidal form. The maximum of the current is shifted to lower values of $\Delta \Phi$ as the temperature is increased until they reach a value of $\Delta \Phi=0.5 \pi$. We track numerically the temperaturedependence of the phase $\chi\left(J_{s}\right)$ where the critical current is reached, and depict the result in Fig.10 (left), where it can be seen that for all three lengths of the ferromagnet, critical currents are reached for phase differences $\Delta \Phi>\pi$ at low temperatures. A temperature-dependence of the critical currents for different lengths of the ferromagnetic block is shown in Fig.10 (right). As expected, we observe that shorter weak links generally increase the critical currents.

\section{CURRENT CONSERVATION}

The Usadel equation entails a spatial conservation law for the Josephson currents. Here, we review that the current is spatially conserved in the presence of the effective self-energy contribution $\hat{\Sigma}(x, \epsilon)$. For this we introduce Keldysh matrices

$$
\check{\mathbb{G}}_{0}=\left(\begin{array}{cc}
\hat{\mathbb{G}}_{0}^{R} & \hat{\mathbb{G}}_{0}^{K} \\
\hat{0} & \hat{\mathbb{G}}_{0}^{A}
\end{array}\right), \quad \hat{\mathbb{G}}_{0}^{K}=\left(\begin{array}{rr}
\mathfrak{G}^{K} & \mathfrak{F}^{K} \\
-\widetilde{\mathfrak{F}}^{K} & -\tilde{\mathfrak{G}}^{K}
\end{array}\right)
$$

where $R, A$, and $K$ refer to retarded, advanced, and Keldysh components, respectively. Similarly, we have

$$
\check{\Delta}=\left(\begin{array}{cc}
\hat{\Delta} & \hat{0} \\
\hat{0} & \hat{\Delta}
\end{array}\right), \quad \check{\Sigma}=\left(\begin{array}{cc}
\hat{\Sigma}^{R} & \hat{\Sigma}^{K} \\
\hat{0} & \hat{\Sigma}^{A}
\end{array}\right) .
$$



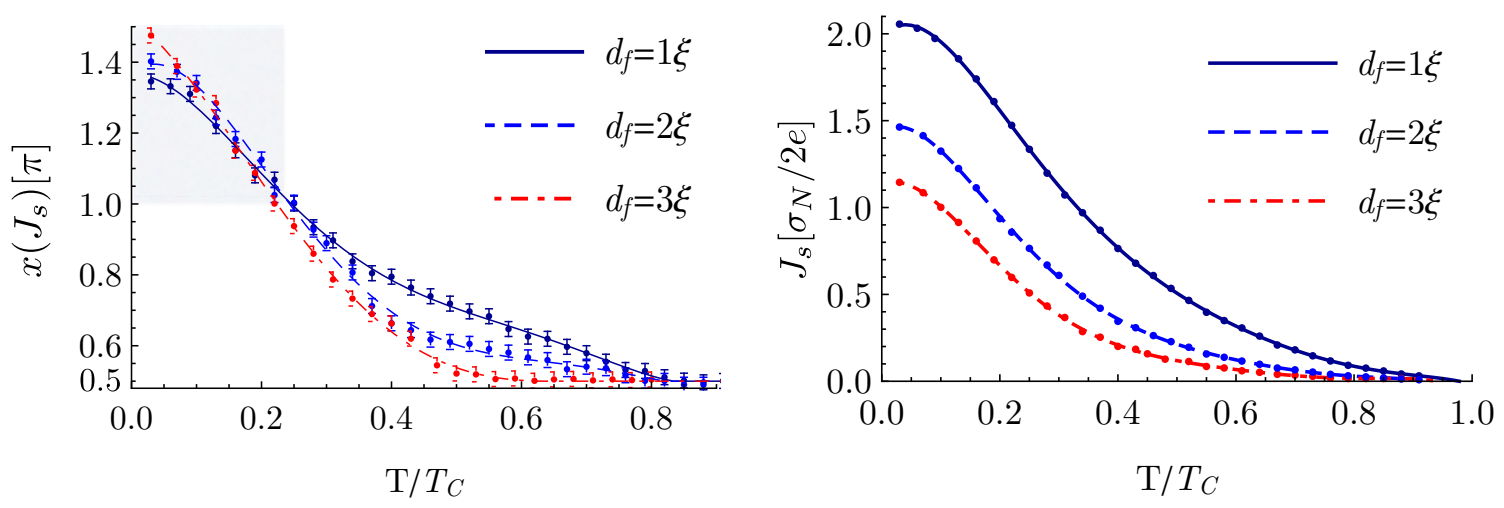

Figure 10: Left: Dots represent the superconducting phase difference at which the maximal (critical) current occurs as function of temperature. At high temperatures the critical current appears at a value of $\pi / 2$ (approximately sinusoidal current-phase relationship), whereas for low temperatures the current-phase relationship is non-sinusoidal. The shaded area indicates the range where the critical current is achieved for $\Delta \Phi>\pi$. The error bars indicate the numerical uncertainty in determining the extrema in the current-phase relationship. Right: Critical current for different system sizes. The current is determined by the maximum of the current-phase relationship Eq. (55). In both plots lines are guides to the eye.

We also define $\check{\tau}_{3}=\mathbb{1}_{2 \times 2} \otimes \hat{\tau}_{3}$. The Usadel equation reads:

$$
\left[\epsilon \check{\tau}_{3}-\check{\Delta}-\check{\Sigma}, \check{\mathfrak{G}}_{0}\right]+\frac{D}{\pi} \partial_{x}\left[\check{\mathbb{G}}_{0} \partial_{x} \check{\mathbb{G}}_{0}\right]=\check{0}
$$

where 0 is an $8 \times 8$ zero matrix. Furthermore, the normalization condition generalizes to

$$
\check{\mathbb{G}}_{0}^{2}=-\pi^{2} \check{1}
$$

where $\breve{1} \equiv \mathbb{1}_{8 \times 8}$ is the $8 \times 8$ unit matrix. This condition is very powerful, as it means that the Keldysh matrix $\check{\mathbb{G}}_{0}$ in Eq. (56) is diagonalizable and its only eigenvalues are $\pm i \pi$.

To derive a current conservation law from the Usadel equation, one has to express the physical current in terms of the Green functions,

$$
J(x)=-e N_{0} \int_{-\infty}^{+\infty} \frac{d \epsilon}{4 \pi} \frac{D}{\pi} \frac{\operatorname{Tr}_{4}}{2}\left(\hat{\tau}_{3}\left[\check{G}_{0}(x, \epsilon) \partial_{x} \check{\mathbb{G}}_{0}(x, \epsilon)\right]^{K}\right)
$$

where $\operatorname{Tr}_{4}$ is a trace over particle-hole and spin space, $e=-|e|$ the charge of the electron, and $N_{0}$ the density of states per spin at the Fermi level in the normal state. The Usadel equation $(58)$ then leads to

$$
-e N_{0} \int_{-\infty}^{+\infty} \frac{d \epsilon}{4 \pi} \frac{\operatorname{Tr}_{4}}{2}\left(\hat{\tau}_{3}\left[\epsilon \check{\tau}_{3}-\check{\Delta}-\check{\Sigma}, \check{\mathbb{G}}_{0}\right]^{K}\right)+\partial_{x} J=0 .
$$

Under cyclic invariance of the trace, the term involving $\epsilon \check{\tau}_{3}$ vanishes immediately:

$$
\epsilon \frac{\operatorname{Tr}_{4}}{2}\left(\left[\check{\mathfrak{G}}_{0}(x, \epsilon)-\check{\tau}_{3} \check{\mathbb{G}}_{0}(x, \epsilon) \check{\tau}_{3}\right]^{K}\right)=0 .
$$

The second term, involving $\check{\Delta}$, vanishes only when $\Delta$ fulfills the self-consistency equation

$$
\Delta(x, T)=\lim _{\epsilon_{c} \rightarrow \infty} \frac{\frac{1}{4 \pi i} \int_{-\epsilon_{c}}^{\epsilon_{c}} d \epsilon \mathfrak{F}^{K}(x, \epsilon, T)}{\int_{-\epsilon_{c}}^{\epsilon_{c}} \frac{d \epsilon}{2 \epsilon} \tanh (\epsilon / 2 T)+\ln \left(T / T_{c}\right)} .
$$

The corresponding contribution in Eq. (61) then reads

$$
\begin{aligned}
& \int_{-\infty}^{+\infty} \frac{d \epsilon}{4 \pi} \frac{\operatorname{Tr}_{4}}{2}\left(\hat{\tau}_{3}\left[\check{\Delta}, \check{\mathfrak{G}}_{0}(x, \epsilon)\right]^{K}\right) \\
& =\lim _{\epsilon_{c} \rightarrow \infty} \int_{-\epsilon_{c}}^{+\epsilon_{c}} \frac{d \epsilon}{4 \pi} \frac{\operatorname{Tr}_{2}}{2}\left(\left\{\mathfrak{F}^{K}, \Delta^{*}\right\}+\left\{\Delta, \tilde{\mathfrak{F}}^{K}\right\}\right)=0
\end{aligned}
$$

where $\operatorname{Tr}_{2}$ is a trace over spin, and Eq. (63) was substituted for $\Delta$ and $\Delta^{*}$, as well as

$$
\tilde{\mathfrak{F}}^{K}(x, \epsilon, T)=\left(\mathfrak{F}^{K}(x,-\epsilon, T)\right)^{*}=-\left(\mathfrak{F}^{K}(x, \epsilon, T)\right)^{\dagger}
$$

used. Due to the cyclic invariance of the trace, the third contribution from the commutator in Eq. (61) vanishes as well:

$$
\int_{-\infty}^{+\infty} \frac{d \epsilon}{4 \pi} \frac{\operatorname{Tr}_{4}}{2}\left(\hat{\tau}_{3}\left[\check{\Sigma}(x, \epsilon), \check{\mathbb{G}}_{0}(x, \epsilon)\right]^{K}\right)=0 .
$$

To proof this, we note that the self energy $\check{\Sigma}$ is proportional to $\check{\mathbb{G}}_{C}$, see Eq. (43), and that, in generalization of Eq. (36),

$$
\left[\check{M}(x, \epsilon), \check{\mathfrak{G}}_{C}(x, \epsilon)\right]=\check{0},
$$


where (see Eq. (37))

$$
\begin{aligned}
\check{M}(x, \epsilon) & =\left(\frac{\mathcal{G}_{q}}{4 \epsilon_{\mathrm{Th}}} \check{\mathfrak{G}}_{\text {Leak }}(\epsilon)+\frac{1}{2} \mathcal{G} \check{\mathfrak{G}}^{F}\right. \\
+ & \left.\frac{1}{2} \mathcal{G}_{P}\left\{\check{\kappa}, \check{\mathfrak{G}}^{F}\right\}-\frac{\pi}{2} \mathcal{G}_{\phi} \check{\kappa}+\frac{1}{2} \mathcal{G}_{S} \check{\mathbb{G}}_{0}(x, \epsilon)\right)
\end{aligned}
$$

where $\check{\mathbb{G}}_{\text {Leak }}=\mathbb{1}_{2 \times 2} \otimes \hat{\mathbb{G}}_{\text {Leak }}, \check{\mathbb{G}}^{F}=\mathbb{1}_{2 \times 2} \otimes \hat{\mathbb{G}}^{F}$, and $\check{\kappa}=\mathbb{1}_{2 \times 2} \otimes \hat{\kappa}$, which all three commute with $\check{\tau}_{3}$. We will assume that $\check{M}$ has distinct eigenvalues (if not, we can always add an infinitesimal term to make them distinct; in fact, it suffices that each characteristic value occurs in only one Jordan block in the Jordan normal form of the matrix). Then, a well-known mathematical theorem ascertains that $\check{\mathbb{G}}_{C}$ can be written uniquely as a polynomial in $\check{M}$ of at most degree 7 (for $8 \times 8$ matrices). Consequently, we can expand $\breve{G}_{C}$ in the following way,

$$
\check{\mathbb{G}}_{C}=\lambda \check{1}+\mu \check{M}+\nu \check{M}^{2}+\rho \check{M}^{3}+\cdots
$$

As $\check{M}$ is of the form $\check{M}=\check{D}+\alpha \check{\mathbb{G}}_{0}$ where $\check{D}$ commutes with $\check{\tau}_{3}$, and because of the condition (59), any power of $\check{M}$ will only have terms that are of the form $\check{D}^{n}$ or $\check{D}^{n} \check{\mathbb{G}}_{0} \check{D}^{m}+\check{D}^{m} \check{\mathbb{G}}_{0} \check{D}^{n}$ or $\sum_{\mathcal{P}[n m k]} \check{D}^{n} \check{\mathbb{G}}_{0} \check{D}^{m} \check{\mathbb{G}}_{0} \check{D}^{k}$ etc. (up to maximally terms containing four $\check{\mathbb{G}}_{0}$ 's), where $\mathcal{P}[n m k]$ means a permutation of $[n m k]$, and $n, m$, and $k$ are integers $\geq 0$. The cyclic property of the trace together with condition (59) then leads to vanishing contributions for each term in Eq. (69) when introduced into Eq. (66) using Eq. (68). For example,

$$
\check{\tau}_{3} \sum_{\mathcal{P}[n m k]}\left(\check{D}^{n} \check{\mathbb{G}}_{0} \check{D}^{m} \check{\mathbb{G}}_{0} \check{D}^{k} \cdot \check{\mathbb{G}}_{0}-\check{\mathbb{G}}_{0} \cdot \check{D}^{n} \check{\mathbb{G}}_{0} \check{D}^{m} \check{\mathbb{G}}_{0} \check{D}^{k}\right)
$$

turns, when using cyclic permutation under the trace and commutation between $\check{D}$ and $\check{\tau}_{3}$, into

$$
\check{\tau}_{3} \sum_{\mathcal{P}[n m k]}\left(\check{D}^{n} \check{\mathbb{G}}_{0} \check{D}^{m} \check{\mathbb{G}}_{0} \check{D}^{k} \check{\mathbb{G}}_{0}-\check{D}^{k} \check{\mathbb{G}}_{0} \check{D}^{n} \check{\mathbb{G}}_{0} \check{D}^{m} \check{\mathbb{G}}_{0}\right),
$$

and as $[k n m]$ is a permutation of $[n m k]$, the two terms cancel when summing over all permutations (we use $\left.\left[\check{\tau}_{3} \check{A}\right]^{K}=\hat{\tau}_{3}[\check{A}]^{K} \equiv \hat{\tau}_{3} \hat{A}^{K}\right)$,

Consequently, collecting all results together, it follows that for our theory

$$
\partial_{x} J(x)=0,
$$

which is the (stationary) charge conservation law.

\section{S-F-S STRUCTURE WITH A MAGNETIC DOMAIN WALL}

We numerically investigate an S-F-S structure that exhibits a magnetic domain wall, see Fig. 11. Such a structure was treated previously in the Ref. 23, and later in Ref. 36. We extend the results in Ref. 23 by (a) calculating the pair potential self-consistently, and by (b)

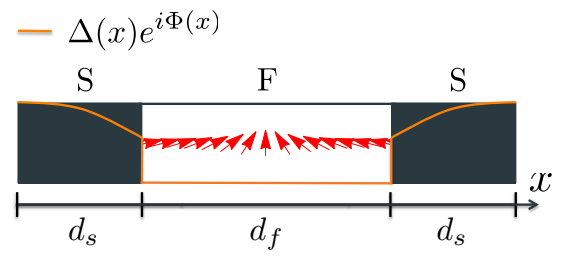

Figure 11: Illustration of the SFS structure. The ferromagnetic block exhibits a non-trivial magnetic domain wall structure. The solid line shows a typical variation of the pairpotential within the structure.

calculating the current-phase relationships as well as the temperature-dependence of the critical currents. In addition, we chose a different, normalized, domain wall parameterization, such that the the magnetization vector $\boldsymbol{J}$ at the start (end) of the ferromagnetic block is always fully polarized in the $+(-) x$-direction. We keep $|\boldsymbol{J}|$ constant and spatially vary the orientation, $\boldsymbol{J} \rightarrow \boldsymbol{J}(x)$, by using the following domain wall parameterization,

$$
\begin{aligned}
& J_{x}=J \cos \left(\frac{\arctan \left(\frac{x-x_{0}}{d_{w}}\right)}{\arctan \left(\frac{d_{s}-x_{0}}{d_{w}}\right)} \frac{\pi}{2}\right), \\
& J_{z}=J \sin \left(\frac{\arctan \left(\frac{x-x_{0}}{d_{w}}\right)}{\arctan \left(\frac{d_{s}-x_{0}}{d_{w}}\right)} \frac{\pi}{2}\right)
\end{aligned}
$$

where $d_{w}$ is the domain wall width, $J_{y}=0.0$ and $x_{0}=d_{s}+d_{f} / 2$ denotes the middle of the S-F-S structure. The ferromagnetic region extends from $x=d_{s}$ to $x=d_{s}+d_{f}$.

We point out that the domain-wall parameterization in Ref. 23 had a fixed rotation pitch given by the domain wall thickness, but independent of the thickness of the ferromagnet layer. $\frac{37}{7}$ Therefore, for higher domain-wall widths the magnetization was already tilted at the interfaces between the ferromagnetic block and the superconductors. Here, we chose a different parameterization by normalizing the argument in the expressions (71)-(72) for the magnetization. This is appropriate for the case that the direction of the magnetic moment at the interfaces is determined by magnetic shape anisotropy.

The transport equation is given by Eq. (\$) with the replacement $\epsilon \rightarrow(\epsilon-\boldsymbol{J} \cdot \boldsymbol{\sigma})$ to account for the spatial variation of the magnetization. In the Riccati parameterization, see Eq. (10) and Eq. (11), the equations read:

$$
\begin{aligned}
& \frac{d^{2} \gamma}{d x^{2}}+\left(\frac{d \gamma}{d x}\right) \frac{\tilde{\mathfrak{F}}}{i \pi}\left(\frac{d \gamma}{d x}\right)= \\
& \frac{i}{D}\left[\gamma \Delta^{*} \gamma-(\epsilon 1-\boldsymbol{J} \cdot \boldsymbol{\sigma}) \gamma-\gamma\left(\epsilon 1+\boldsymbol{J} \cdot \boldsymbol{\sigma}^{*}\right)-\Delta\right] \\
& \frac{d^{2} \tilde{\gamma}}{d x^{2}}+\left(\frac{d \tilde{\gamma}}{d x}\right) \frac{\mathfrak{F}}{-i \pi}\left(\frac{d \tilde{\gamma}}{d x}\right)= \\
& \frac{-i}{D}\left[\tilde{\gamma} \Delta \tilde{\gamma}+(\epsilon 1+\boldsymbol{J} \cdot \boldsymbol{\sigma}) \tilde{\gamma}+\tilde{\gamma}(\epsilon 1-\boldsymbol{J} \cdot \boldsymbol{\sigma})-\Delta^{*}\right]
\end{aligned}
$$




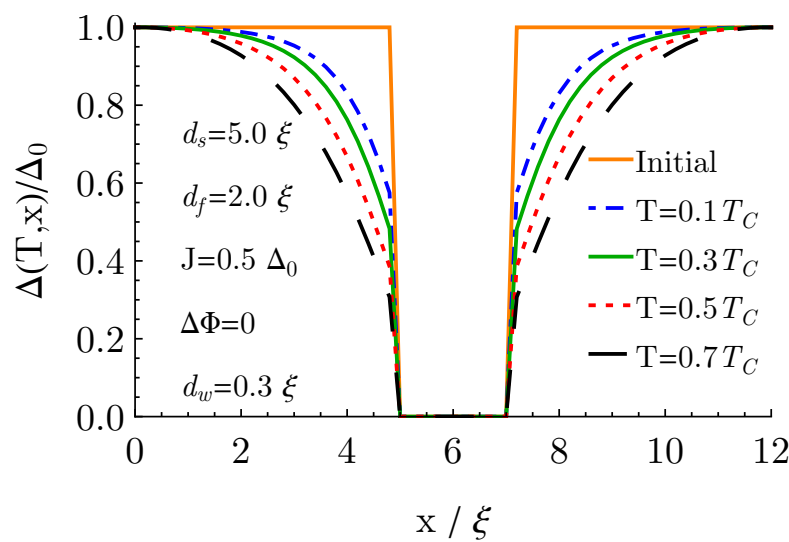

Figure 12: Suppression of the pair potential for several temperatures illustrated for an S-F-S structure without phase gradients. The solid line (Initial) is the step-like pair potential without a self-consistent calculation, depicted for comparison.

At the $S / F$ interfaces $\left(x_{i}^{S}, x_{i}^{F}\right)$ we connect the $\gamma, \tilde{\gamma}$ by continuity conditions: $\frac{23}{}$

$$
\begin{aligned}
\gamma\left(x_{i}^{S}\right) & =\gamma\left(x_{i}^{F}\right) \\
\frac{d}{d x} \gamma\left(x_{i}^{S}\right) & =\frac{d}{d x} \gamma\left(x_{i}^{F}\right) .
\end{aligned}
$$

The pair potential $\Delta(T, x)$ is calculated self-consistently according to Eq. 48). This takes into account the suppression of the order parameter close to the ferromagnetic material (inverse proximity effect). In Fig. 12 we show the typical behavior of the order parameter as the temperature of the system is varied for a ferromagnet that hosts a domain wall.

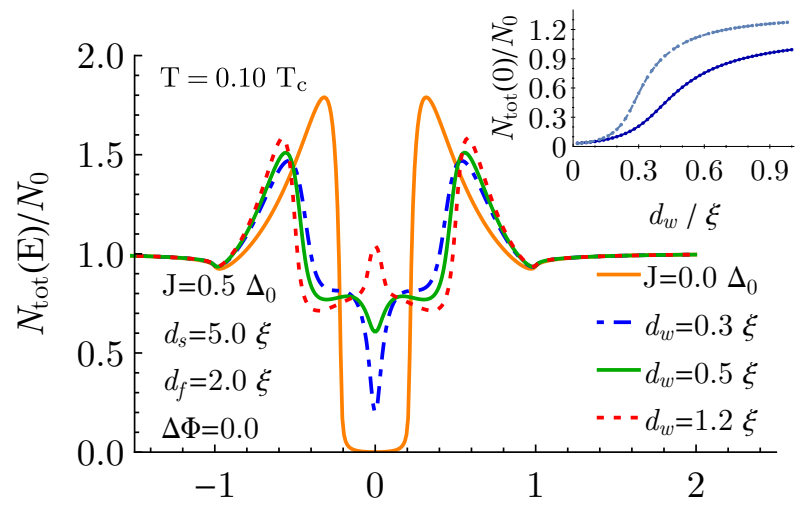

$\mathrm{E} / \Delta$

Figure 13: Self-consistent LDOS, $N_{\text {tot }}$, as a function of energy $E$, normalized to its normal state value $N_{0}$, for several domain wall widths $d_{w}$ calculated in the middle of the $F$ region in an S-F-S structure. The case of an S-N-S structure $(J=0)$ is shown for comparison as a solid line. Inset: $N_{\text {tot }}$ at the chemical potential $(E=0)$ as function of domain wall width $d_{w}$ for self-consistent pair potential (full line) and for nonself-consistent (step-like) pair potential (dashed lines).
The effect of the domain wall on the local density of states in the system can be seen in Fig. 13. In comparison to the normal metal $(J=0)$ the minigap is populated with additional Andreev bound states that stem from spin-triplet correlations that are sensitive to the direction of the magnetization. Non-zero $J_{x}$ and $J_{z}$ components convert singlet into triplet amplitudes ${ }^{[7} \mathrm{A}$ non-vanishing $J_{x}$ induces spin-flips and breaks up a spinsinglet Cooper pair and converts it into an unequal spintriplet state $\sim(|\uparrow \uparrow\rangle-|\downarrow \downarrow\rangle)$ whereas $J_{z}$ induces equal spin-triplet pairings $\sim(|\uparrow \downarrow\rangle+|\downarrow \uparrow\rangle)$. In the case of increasing domain wall widths, the magnetic domain wall encourages such spin-flip processes and thus creates new Andreev bound states. As the domain wall width increases, spectral weight from the shoulders fills up the minigap, as illustrated in Fig. 13. The inset of Fig. 13 shows the value of the local density of states at the chemical potential as function of domain wall width. There is a characteristic value $d_{w}^{*}$ at which a step-like feature occurs in this plot. In comparison to the case of a non-selfconsistent pair potentia 23 , shown as dashed line, this characteristic value $d_{w}^{*}$ is shifted upwards. When the magnetic domain wall extends over the whole ferromagnetic region, $J$ varies slowly with $x$. This case is similar to the case for a fully polarized ferromagnet. The minigap thus vanishes and local minima appear at approximately $\pm J / \Delta$. The same effect can be observed for the (S-(F|S)-S) structure, where the local density of states is spin-split, see Fig.7.

For a given domain wall width, we investigate the dependence of the local density of states on the applied phase gradient, see Fig. 14. When a finite phase differ-

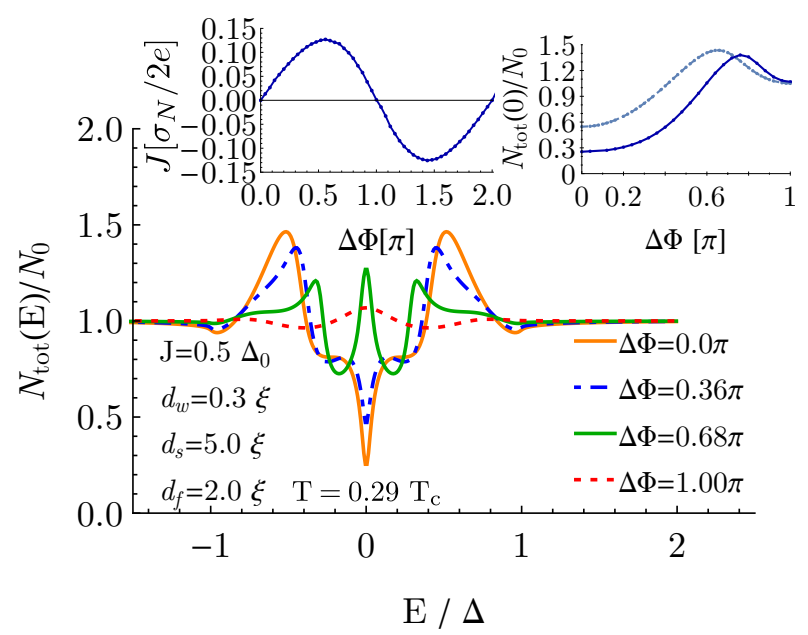

Figure 14: Self-consistent LDOS, $N_{\text {tot }}(E)$, in an S-F-S structure with domain wall as a function of energy for several phase differences $\Delta \Phi$ between the superconductors, calculated in the middle of the $F$ region, and normalized to the normal state value $N_{0}$. Left inset: current-phase relationship. Right inset: $N_{\text {tot }}$ at the chemical potential, $E=0$. Full line for self-consistent pair potential. The dashed line shows for comparison the result for a non-self-consistent pair potential. 
ence is present at the outer elements, supercurrents can flow in the S-F-S-structure. A finite phase difference $\Delta \Phi$ modifies the local density of states as it adds to the phase that is picked up by the quasiparticles during the diffusive motion through the ferromagnet. In particular the zero-energy density of states is influenced strongly by the applied phase difference, as it can be seen in the right inset of Fig. 14. It increases smoothly until a maximum value for $\Delta \Phi<\pi$ is reached. The plot is mirror symmetric around $\Delta \Phi=\pi$ (only values for $\Delta \Phi<\pi$ are shown). For comparison we also reproduce the non-self-consistent result of Ref. 23 as a dashed line in the inset. We observe that self-consistency of the order parameter gives pronounced corrections to the local density of states, in particular its value at the chemical potential. Experimentally, tunnel current measurements provide access to the zero-energy density of states.

We also present here self-consistent supercurrents in the S-F-S structure. Supercurrents have not been studied in Ref. 23. In Fig. 15 we plot the temperaturedependence of the critical currents for both a S-N-S structure (full lines) and a S-F-S structure that hosts a domain wall (dashed lines). Additionally, we numerically track the temperature-dependence of the phase difference that leads to the critical current, see inset in Fig. 15. The critical currents in the S-F-S structure are lowered by a magnetic domain wall in comparison to the case when a magnetic structure is missing, such as is the case in a S-N$\mathrm{S}$ structure. The current-phase relationship in both cases becomes sinusoidal at high temperatures, where the maximum current is reached at a phase difference $\Delta \Phi=\pi / 2$. This is reflected in the critical currents as well, as the curves for the S-N-S and the S-F-S structure collapse onto each other approximately when for both cases a sinusoidal current-phase relationship is established. In the

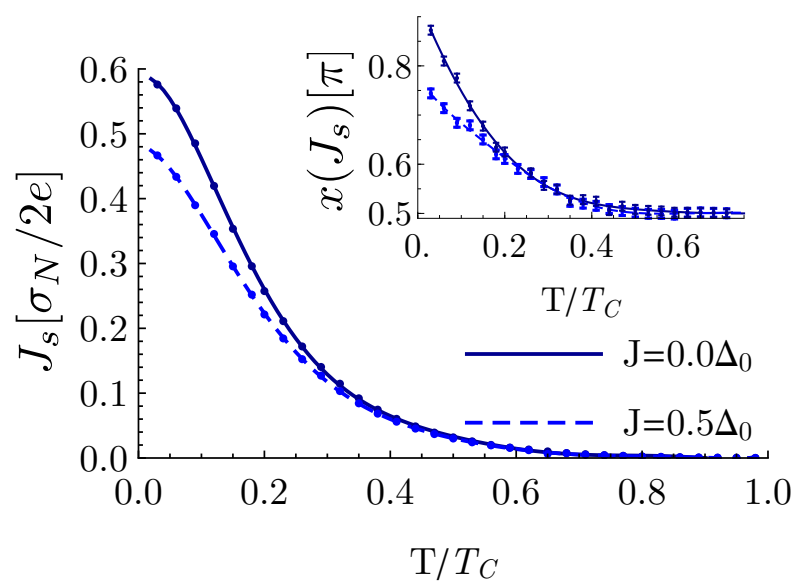

Figure 15: Critical Josephson currents for the parameters $d_{s}=5.0 \xi, d_{f}=2.0 \xi$, and a domain-wall of length $d_{w}=0.3 \xi$. The inset shows the phases for which the maximal (critical) current is reached. The error bars indicate the numerical uncertainty in determining the extrema in the current-phase relationship. Lines are guides to the eye. low-temperature-regime, the critical currents in the S-N$\mathrm{S}$ structure are offset to higher values than in the case for the S-F-S structure with a domain wall. In both structures however the maximum current is achieved for phase gradients $\Delta \Phi<\pi$, see the inset of Fig. 15. This should be compared to the $\mathrm{S}-(\mathrm{F} \mid \mathrm{S})-\mathrm{S}$ structure where critical currents are reached for phase differences $\Delta \Phi>\pi$.

\section{CONCLUSION}

Using the model for an S-(S|F)-S Josephson junction depicted in Fig. 1, we have transformed spin-dependent boundary conditions within the $(\mathrm{S} \mid \mathrm{F})$ bilayer into an effective self energy that enters the Usadel transport equation. This allows for a numerically very effective handling of the transport equation. We have used our model to calculate important measurable quantities such as the density of states, spin-magnetizations, the pair potential, and the critical Josephson currents through the system. We also proved that our theory explicitly fulfills the continuity equation, expressing charge conservation, provided self-consistently determined order parameter profiles are used.

We have in particular studied the weak link behavior of such an S- $(\mathrm{S} \mid \mathrm{F})-\mathrm{S}$ Josephson junction, showing the characteristic hysteretic current-phase relation ${ }^{3}$, as indicated by a multi-valued solution. In our case the suppression of superconducting order in the weak-link region is achieved via proximity coupling to a strongly spin-polarized ferromagnet. We study long weak-link structures with a length comparable or larger than the superconducting coherence length. We present a detailed quantitative solution for this problem. We find that self-consistency of the order parameter profile across the weak link is necessary in order to be able to determine the Josephson current in a sensible way.

We also consider a second geometry, an S-F-S junction in which a magnetic domain wall is situated in the center of the $\mathrm{F}$ region. We have extended previous work $23 \mid 38,45$ by studying in particular the effect of self consistency of the order parameter in the superconducting leads. We find that self-consistency of the order parameter leads to pronounced modification of the results, in particular the functional dependence of the density of states on domain wall width. We also calculated the critical Josephson current and find that it is considerably reduced at low temperatures by the presence of a domain wall.

\section{Acknowledgments}

J.G. acknowledges financial support by SEPnet/GRADnet during his Euromasters study at Royal Holloway, University of London. J.G. and M.E. appreciate the stimulating atmosphere within the Hubbard Theory Consortium. M.E. acknowledges support by EPSRC (Grant No. EP/J010618/1 and EP/N017242/1). 
1 Yu. A. Izyumov, Yu. N. Proshin, and M. G. Khusainov, Usp. Fiz. Nauk 172, 113-154 (2002), Конкуренция сверхпроводимости и магнетизма в гетероструктурах ферромагнетик/сверхпроводник, Engl. transl.: Phys. Usp. 45 109-148 (2002), Competition between superconductivity and magnetism in ferromagnet/superconductor heterostructures

${ }^{2}$ M. Eschrig, J. Kopu, A. Konstandin, J. C. Cuevas, M. Fogelström, and G. Schön, Advances in Solid State PhysicsPergamon Press THEN Vieweg- 44, 533-546 (2004), Singlet-triplet mixing in superconductor-ferromagnet hybrid devices

3 Golubov, A. A. and Kupriyanov, M. Yu. and Il'ichev, E., Rev. Mod. Phys. 76, 411-469 (2004), The current-phase relation in Josephson junctions

4 F.S. Bergeret, A.F. Volkov, and K.B. Efetov, Rev. Mod. Phys. 77, 1321-1373 (2005), Odd triplet superconductivity and related phenomena in superconductor-ferromagnet structures

5 A. I. Buzdin, Rev. Mod. Phys. 77, 935-976 (2005), Proximity effects in superconductor-ferromagnet heterostructures

6 I. F. Lyuksyutov and V. L. Pokrovsky, Adv. Phys. 54, 67136 (2007), Ferromagnet-superconductor hybrids

7 M. Eschrig, Physics Today 64, 43-49 (2011), Spin-polarized supercurrents for spintronics

8 M. G. Blamire and J. W. A. Robinson, J. Phys. Condens. Matter 26 453201-(1-13) (2014), The interface between superconductivity and magnetism: understanding and device prospects

9 M. Eschrig, Reports on Progress in Physics 78, 104501 (2015), Spin-polarized supercurrents for spintronics: a review of current progress

10 J. Linder and J. W. A. Robinson, Nature Physics 11, 307315 (2015), Superconducting spintronics

11 P. Machon, M. Eschrig, and W. Belzig, Phys. Rev. Lett. 110, 047002-(1-5) (2013), Nonlocal Thermoelectric Effects and Nonlocal Onsager relations in a Three-Terminal Proximity-Coupled Superconductor-Ferromagnet Device

12 S. Kawabata, A. Ozaeta, A. S. Vasenko, F. W. J. Hekking, and F. S. Bergeret, Appl. Phys. Lett. 103, 032602 (2013), Efficient electron refrigeration using superconductor/spinfilter devices

13 P. Machon, M. Eschrig, and W. Belzig, New Journal of Physics 16, 073002-(1-19) (2014), Giant thermoelectric effects in a proximity-coupled superconductor-ferromagnet device

14 A. Ozaeta, P. Virtanen, F. S. Bergeret, and T. T. Heikkilä, Phys. Rev. Lett. 112, 05700 (2014), Predicted Very Large Thermoelectric Effect in FerromagnetSuperconductor Junctions in the Presence of a SpinSplitting Magnetic Field

15 F. Giazotto, P. Solinas, A. Braggio, and F. S. Bergeret, Phys. Rev. Applied 4, 044-16 (2015), FerromagneticInsulator-Based Superconducting Junctions as Sensitive Electron Thermometers

16 T. Yu. Karminskaya and M. Yu. Kupriyanov, Pis'ma Zh. Eksp, Teor. Fiz. 85, 343-348 (2007), Эфбективное уменъшение обменной энергии в $S$-(FN)-S джсоефсоновских структурах, Engl. transl.: JETP Lett. 85, 286281 (2007), Effective Decrease in the Exchange Energy in $S-(F N)-S$ Josephson Structures; Pis'ma Zh. Eksp, Teor.
Fiz. 86, 65-70 (2007), Переход из 0 в $\pi$-состояние в $S$-(FNF)-S джсозефсоновских структурах, Engl. transl.: JETP Lett. 86, 61-66 (2007), Transition from the 0 State to the $\pi$ State in $S-F N F-S$ Josephson Structures

17 T. E. Golikova, F. Hübler, D. Beckmann, I. E. Batov, T. Yu. Karminskaya, M. Yu. Kupriyanov, A. A. Golubov, and V. V. Ryazanov, Phys. Rev. B 86, 064416-(1-5) (2012), Double proximity effect in hybrid planar Superconductor(Normal metal/Ferromagnet)-Superconductor structures

18 T. Yu. Karminskaya, A. A. Golubov, M. Yu. Kupriyanov, and A. S. Sidorenko, Phys. Rev. B 79, 214509-(1-13) (2009), Josephson effect in superconductor/ferromagnetnormal/superconductor structures; Phys. Rev. B 81, 214518-(1-13) (2010), Josephson effect in superconductor/ferromagnet structures with a complex weak-link region

19 J. A. X. Alexander, T. P. Orlando, D. Rainer, and P. M. Tedrow, Phys. Rev. B 31 5811-25 (1985) Theory of Fermiliquid effects in high-field tunneling

20 T. Tokuyasu, J. A. Sauls, and D. Rainer, Phys. Rev. B 38, 8823-8833 (1988), Proximity effect of a ferromagnetic insulator in contact with a superconductor

21 F. S. Bergeret, A. F. Volkov, and K. B. Efetov Europhys. Lett. 66111 (2004), Spin screening of magnetic moments in superconductors; Phys. Rev. B 69 174504, (2004), Induced ferromagnetism due to superconductivity in superconductorferromagnet structures

22 K. D. Usadel, Phys. Rev. Lett. 25, 507-509 (1970), Generalized Diffusion Equation for Superconducting Alloys

23 A. Konstandin, J. Kopu, and M. Eschrig, Phys. Rev. B 72, 140501-(1-4)(R) (2005), Superconducting proximity effect through a magnetic domain wall

24 J. C. Cuevas, J. Hammer, J. Kopu, J. K. Viljas, and M. Eschrig, Phys. Rev. B 73, 184505-(1-6) (2006), Proximity effect and multiple Andreev reflections in diffusive superconductor-normal-metal-superconductor junctions

25 M. Eschrig, A. Cottet, W. Belzig, and J. Linder, New Journal of Physics 17, 083037-(1-21) (2015), General Boundary Conditions for Quasiclassical Theory of Superconductivity in the Diffusive Limit: Application to Strongly Spin-polarized Systems

26 A. Cottet, D. Huertas-Hernando, W. Belzig, and Yu. V. Nazarov, Phys. Rev. B 80, 184511-(1-17) (2009) [Erratum: Phys. Rev. B 83, 139901 (2011)], Spin-dependent boundary conditions for isotropic superconducting Green's functions

27 W. Belzig, F. K. Wilhelm, C. Bruder, G. Schön, and A. D. Zaikin, Superlattices Microstruct. 25 1251-1288 (1999), Quasiclassical Green's function approach to mesoscopic superconductivity

28 G. Eilenberger, Z. Phys. 214, 195-213 (1968) Transformation of Gor'kov's equation for type II superconductors into transport-like equations

29 A. I. Larkin and Yu. N. Ovchinnikov, Zh. Eksp. Teor. Fiz. 55, 2262-2272 (1968), О квазиклассическом методе в теории сверхпроводимости, Engl. transl.: Sov. Phys.-JETP 28, 1200-1205 (1969), Quasiclassical Method in the Theory of Superconductivity

30 M. Eschrig, Phys. Rev. B 61, 9061-9076 (2000), Distribution functions in nonequilibrium theory of superconductivity and Andreev spectroscopy in unconventional superconductors

31 A. Brataas, Yu. V. Nazarov, and G. E. W. Bauer, Phys. 
Rev. Lett. 11, 2481-2484 (2000). Finite-Element Theory of Transport in Ferromagnet-Normal Metal Systems

${ }^{32}$ Yu. V. Nazarov, Superlatt. and Microstruc. 25, 1221-1231 (1999), Novel circuit theory of Andreev reflection

${ }_{3}$ Yu. V. Nazarov, Phys. Rev. Lett. 73, 1420 (1994), Circuit Theory of Andreev Conductance

${ }^{34}$ T. Champel and M. Eschrig, Phys. Rev. B 72, 054523(1-11) (2005), Effect of an inhomogeneous exchange field on the proximity effect in disordered superconductorferromagnet hybrid structures

35 A. F. Andreev, Zh. Eksp. Teor. Fiz. 46, 1823-1828 (1964), Теплопроводность промежуточного состояния сверхпроводников, Engl. transl.: Sov. Phys. JETP 19, 12281231 (1964), Thermal conductivity of the intermediate state of superconductors

36 T. Champel, T. Löfwander, and M. Eschrig Phys. Rev. Lett. 100, 077003 (2008), 0- $\pi$ transitions in a superconductor/chiral ferromagnet/ superconductor junction induced by a homogeneous cycloidal spiral

37 We note here a typo in Eq. 10 of Ref. 23, where it should $\operatorname{read} \theta(z)=-\arctan \left(4 \frac{z-z_{0}}{d_{W}}\right)$.

${ }^{38}$ F. S. Bergeret, A. F. Volkov, and K. B. Efetov Phys. Rev. Lett. 86, 4096 (2001) Long-range proximity effects in superconductor-ferromagnet structures

39 A. F. Volkov, A. Anishchanka, and K. B. Efetov Phys. Rev.
B 73, 104412 (2006), Odd triplet superconductivity in a superconductor/ferromagnet system with a spiral magnetic structure

40 A. F. Volkov and K. B. Efetov Phys. Rev. B 78, 024519 (2008), Odd triplet superconductivity in a superconductor/ferromagnet structure with a narrow domain wall

41 J. Linder, T. Yokoyama, and A. Sudbø Phys. Rev. B 79, 054523 (2009), Theory of superconducting and magnetic proximity effect in $S / F$ structures with inhomogeneous magnetization textures and spin-active interfaces

42 M. Alidoust, J. Linder, G. Rashedi, T. Yokoyama, and A. Sudbø, Phys. Rev. B 81, 014512 (2010), Spin-polarized Josephson current in superconductor/ferromagnet/superconductor junctions with inhomogeneous magnetization

43 A. I. Buzdin, A. S. Melnikov, and N. G. Pugach, Phys. Rev. B 83, 144515 (2011), Domain walls and long-range triplet correlations in SFS Josephson junctions

44 C.-T. Wu, O. T. Valls, and K. Halterman, Phys. Rev. Lett. 108, 117005 (2012), Reentrant superconducting phase in conical-ferromagnetsuperconductor nanostructures

45 J. Linder and K. Halterman Phys. Rev. B 90 104502, (2014), Superconducting spintronics with magnetic domain walls 